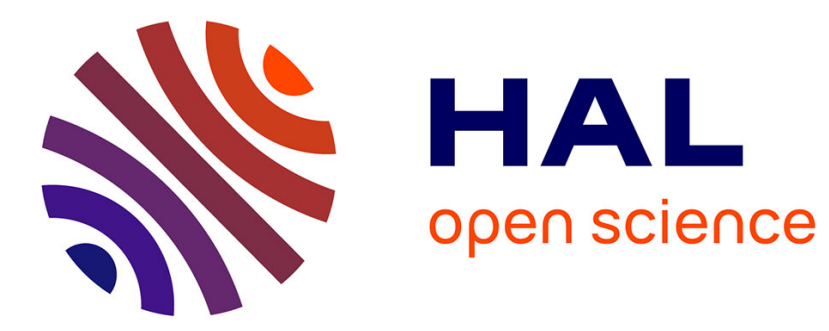

\title{
Scaling effect in notched composites: The Discrete Ply Model approach
}

\author{
Joël Serra, Christophe Bouvet, Bruno Castanié, Caroline Petiot
}

\section{To cite this version:}

Joël Serra, Christophe Bouvet, Bruno Castanié, Caroline Petiot. Scaling effect in notched composites: The Discrete Ply Model approach. Composite Structures, 2016, 148, pp.127-143. 10.1016/j.compstruct.2016.03.062 . hal-01828731

\section{HAL Id: hal-01828731 https://hal.science/hal-01828731}

Submitted on 3 Jul 2018

HAL is a multi-disciplinary open access archive for the deposit and dissemination of scientific research documents, whether they are published or not. The documents may come from teaching and research institutions in France or abroad, or from public or private research centers.
L'archive ouverte pluridisciplinaire HAL, est destinée au dépôt et à la diffusion de documents scientifiques de niveau recherche, publiés ou non, émanant des établissements d'enseignement et de recherche français ou étrangers, des laboratoires publics ou privés. 


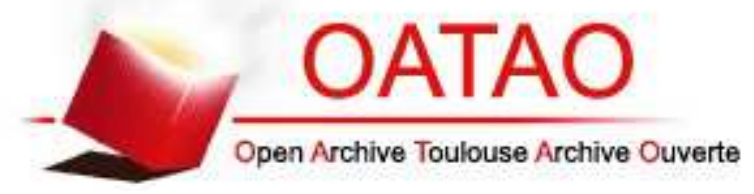

\section{Open Archive TOULOUSE Archive Ouverte (OATAO)}

OATAO is an open access repository that collects the work of Toulouse researchers and makes it freely available over the web where possible.

This is an author-deposited version published in : http://oatao.univ-toulouse.fr/ Eprints ID : 15705

To link to this article: DOI:10.1016/j.compstruct.2016.03.062

URL: http://dx.doi.org/10.1016/j.compstruct.2016.03.062

To cite this version: Serra, Joël and Bouvet, Christophe and Castanié, Bruno and Petiot, Caroline Scaling effect in notched composites: The Discrete Ply Model approach. (2016) Composite Structures, vol. 148. pp. 127-143. ISSN 0263-8223

Any correspondence concerning this service should be sent to the repository administrator: staff-oatao@listes-diff.inp-toulouse.fr 


\title{
Scaling effect in notched composites: The Discrete Ply Model approach
}

\author{
Joël Serra ${ }^{a}$, Christophe Bouvet ${ }^{\mathrm{a}}$, Bruno Castanié ${ }^{\mathrm{a}, *}$, Caroline Petiot ${ }^{\mathrm{b}}$ \\ ${ }^{a}$ Groupe Matériaux et Structures Composites (MSC), Université de Toulouse, FRE CNRS 3687, INSA/UPS/ISAE/Mines Albi, Institut Clément Ader, 3 Rue Caroline Aigle, \\ 31400 Toulouse, France \\ ${ }^{\mathrm{b}}$ Airbus Group Innovations, 12, rue Pasteur, 92152 Suresnes, France
}

Keywords:

Size effect

Open-hole tensile test

Discrete Ply Modeling

Experiments

Finite element model

\begin{abstract}
A B S T R A C T
Numerical and experimental investigations were carried out on the size effect in notched carbon/epoxy laminates. This paper presents a computational study of scaled open-hole tensile tests using the Discrete Ply Modeling (DPM) method, which has already proven efficient on both in-plane and out-of-plane loading cases, such as pull through, low velocity impact and compression after impact. The specificities of this finite element model are its discrete nature, the small number of parameters required and its robustness. Three different stacking sequences of thin plies coupled with three sizes of coupons having the same length to width ratio were tested. The results show that the model reflects the reduction in strength when the size of the specimen increases. The influence of different parameters such as mesh size, presence of discrete matrix cracks and fiber fracture toughness that should be used for clustered plies, are discussed. Comparisons with experiments demonstrate that tensile strengths, and failure scenarios and patterns are predicted with acceptable accuracy.
\end{abstract}

\section{Introduction}

Composite materials have now become indispensable for most transport vehicles, especially in the aerospace and astronautics sectors [1]. The assembly of structures using such materials cannot avoid the presence of holes or cut-outs, which induce stress concentrations and reduce strength. Notched strength is hence one of the design drivers for composite structures. Moreover, holes have the particularity that they can be employed to model other complex forms of damage such as impacts or through-thethickness cracks [2]. Scaling effects are at least as important since most tests are carried out on small coupons whereas real structures are 10-100 times larger. A deeper understanding of these scaling phenomena is still required in spite of the substantial amount of research devoted to it since its discovery by Leonardo da Vinci in the early 1500s [3].

Numerous experiments have been conducted to explore the physical causes of these phenomena [4-6]. As detailed in [7], size effects can occur at different levels. At the material level, an influence of the thickness has been detected, called the "in situ" effect. This phenomenon has been analyzed by Wisnom et al. on isotropic specimens in [5]. At the structural level, it has been proven that the

\footnotetext{
* Corresponding author.

E-mail address: bruno.castanie@insa-toulouse.fr (B. Castanié).
}

strength of notched composite laminates decreases with increasing notch sizes when thin plies are used. It is the latter scale that is the focus of this article. The "hole size effect" is triggered by the presence of non-critical ply-level damage such as fiber fracture, fiber splitting, delamination and matrix failure in the vicinity of the hole [8], which blunts the stress concentration. The extent of this "fracture process zone" relative to the size of the specimen explains the strength difference between small and large specimens [7].

Preliminary sizing solutions were found with the point stress or the average stress models $[9,10]$ and all their extensions described by Awerbuch and Madhukar [11] or, more recently, with a volume based criterion developed by Hochard et al. [12]. Camanho et al. then proposed an alternative method for predicting the strength of composite laminates loaded in tension and containing holes or cracks [13]. This analytical model, based on finite fracture mechanics and first introduced by Leguillon [14], predicts failure when both stress-based and energy-based criteria are satisfied. Mohammed et al. used two-parameter cohesive laws [15] to determine the strength of an open-hole specimen. This analytical work, based on the original cohesive law concept of Dugdale [16] and Barenblatt [17] made it possible to link the failure strength to the length of the failure processing zone, and this length at failure load to the specimen dimension and geometry. Good agreement between experimental data and numerical predictions was obtained with these preliminary sizing methods for quasi isotropic laminates. 
However, experimental tests revealed the substantial influence of the stacking sequence on both failure scenarios and strengths $[8,18,19]$. For example, the final failure of laminates manufactured with thick plies is mainly due to delamination [5]. In our study, it was experimentally determined on plain specimens that failure strength varied by thirty percent between two stacking sequences of a laminate containing the same number of plies in each direction. These fast semi-analytical and numerical methods do not take the influence of the stacking sequence into account since they consider that strain is constant within the laminate thickness [20]. This is why a more complete numerical model is needed to predict the physics associated with these failure phenomena (delamination and transverse cracks). Following the "Virtual Testing" approach, i.e. moving from numerous, expensive experimental tests towards robust, accurate numerical simulations, a large number of models have been proposed to fit experimental data.

Some of these numerical studies of size effects on open-hole tensile composite laminates have already been described by Chen et al. [22]. They can be classified according to their level of discretization [21], from the whole continuum model without any interfaces to the most discretized type of model where the three most important types of damage (fiber failure, matrix cracking and delamination) are all represented through interfaces (Fig. 1). The latter type of model has not been developed yet, probably because of its prohibitive calculation cost. Among the models available for the "Level 1" category is the model by Camanho et al. [7] which, based on continuum damage mechanics, can predict damage onset and the extent and type of non-critical damage mechanisms, without any calibration. Abisset et al. also employ a damage mesomodel based on a continuum mechanics approach. Diffuse damage and transverse cracking are modeled with a progressive evolution law, whereas a brittle evolution law is used for fiber breakage [23]. This model has been used to predict the main features of the three failure modes of a notched specimen subjected to tensile stress. However, it is important to mention that it requires a large number of parameters to identify. "Level 2 " refers to models where the delamination is modeled using discrete elements. Pinho developed a model that predicts fiber and matrix failures using smeared crack models and delamination using cohesive interfaces [22]. ONERA developed a model composed of a multi-scale progressive failure approach to describe the softening behavior of a ply failing in fiber mode and cohesive zone elements to model delamination [24]. Similarly, a physics based, progressive damage model has also been developed by
Ridha et al. to represent the different damage mechanisms. The modeling strategy [25] is based on a continuum damage mechanism for in-plane damage progression and cohesive elements for delamination. Discrete elements may also be used to represent matrix cracks. For example, Wisnom and al. use a Weibull approach to predict fiber failure, cohesive elements between plies to model delamination, and within each ply to model potential splits initiating tangentially to the hole $[5,26,27]$. Recent discussions have concerned the need for discrete elements to correctly model matrix cracks and their interaction with delamination [28]. Pioneering this approach, the Discrete Ply Model (DPM) used in this paper employs cohesive interfaces to model matrix failure and delamination, and 3D volume elements to predict fiber failure. It should be noted that other researchers have simulated single matrix cracks using the cohesive zone model approach [29-33] but, as far as the authors know, none of them have focused on the open-hole tensile test scaling effect.

This discrete method (DPM) was initiated by Bouvet et al. for the modeling of low velocity impacts in composite panels [34] and was enhanced afterwards to capture permanent indentation [35] and to simulate compression after impact [36]. In this model, coupling between the intra- and inter-laminar damage is naturally taken into account through the use of interfaces connected by a specific mesh. This model has also been used to represent pullthrough cases [37], where the effects of splitting on the load redistribution were correctly predicted. Moreover, after a modification of the hole contour mesh, the DPM has been able to predict notched strength, and failure scenarios and patterns with reasonable accuracy on different stacking sequences [38].

In this paper, open-hole tensile tests are performed and analyzed by means of the DPM method. Three different stacking sequences of thin plies coupled with three sizes of coupons with the same length to width ratio were tested. The following section gives details of the tests and samples. Then, the DPM modeling strategy is detailed and the results are compared with experimental data. Failure scenarios are analyzed and modeling choices are discussed.

\section{Experimental work}

\subsection{Material and setup}

The material investigated in the present study was Hexcel's T700-M21 carbon epoxy unidirectional tape with a nominal ply thickness of $0.125 \mathrm{~mm}$. Three types of stacking sequences were

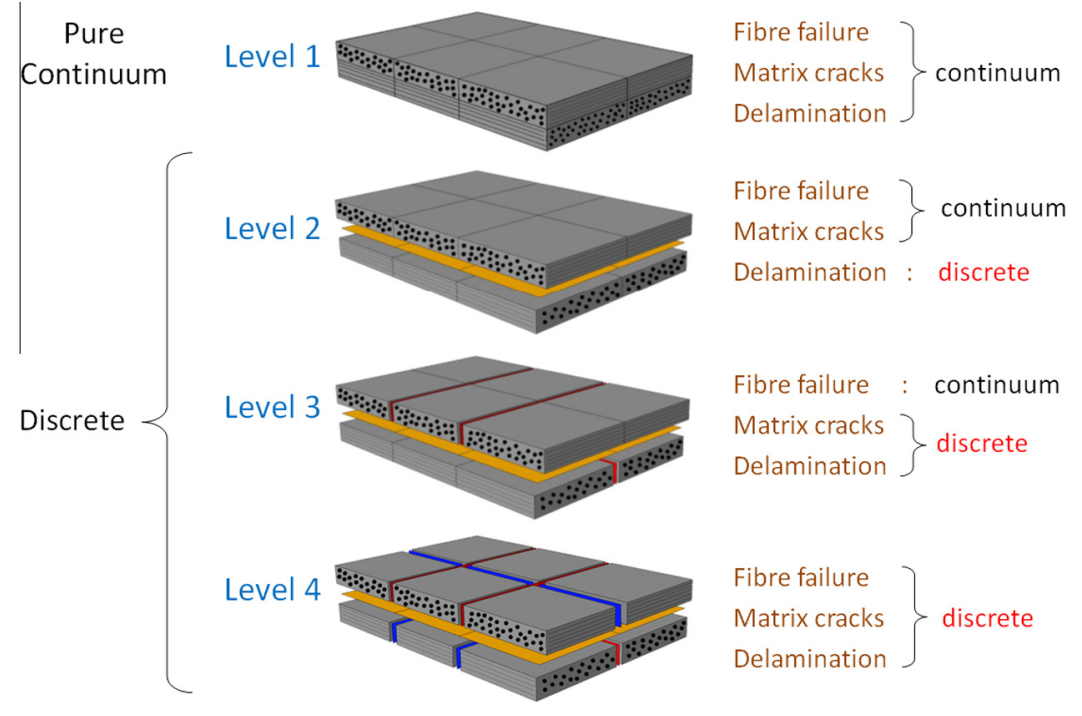

Fig. 1. Numerical damage models architectures [21]. 
studied. Each laminate was symmetric and contained 13 plies, with the same number in each direction $\left(0^{\circ}, 90^{\circ}\right.$ and $\left.\pm 45^{\circ}\right)$, only the relative positions changed from one layup to another:

- C3-1 [45/-45/X/X/X/90/0/90/X/X/X/-45/45]

- C3-2 [X/X/X/X/0/90/0/90/0/X/X/X/X]

- C3-3 [X/X/X/X/X/0/0/0/X/X/X/X/X]

The layups cannot be fully disclosed in this article. The in-plane scaled center notched specimens and plain specimens are shown diagrammatically in Fig. 2. Three different sizes for the notched coupons were tested as described in Table 1 . Specimens with a $1 \mathrm{~mm}$ diameter hole, specimens with a $3.175 \mathrm{~mm}$ diameter hole and specimens with a $6.35 \mathrm{~mm}$ diameter hole are respectively termed "Small", "Medium" and "Large" specimens hereafter.

Tests were performed using a $450 \mathrm{kN}$ SCHENCK hydraulic driven machine for "Medium" and "Large" specimens, a $10 \mathrm{kN}$ INSTRON electromechanical machine for the "Small" and a $20 \mathrm{kN}$ INSTRON electromechanical machine for the "Plain" specimens, at ambient temperature and humidity. Coupons were tested under displacement control with scaled loading rates with regards to the specimen gauge lengths, the value being $0.02 \mathrm{~mm} / \mathrm{s}$ for the "Large" specimens in order to obtain quasi-static loading conditions. The machines provided measurements of displacement and effort. For a more accurate value of the displacement, a linear displacement sensor (LVDT) was used. Notched tests were also monitored by two stereocorrelation cameras on one side of the specimen and an infrared camera on the other as represented in Fig. 3. Image analyses were performed with VIC $3 \mathrm{D}^{\circledR}$ for the stereo results and ALTAIR $^{\circledR}$ for the infrared. It was, however, found after the tests that the coupons had slipped in the machine clamps. Consequently, the strain values shown in this paper come from the displacements determined using image correlation at a distance of 2D from the hole and averaged over a distance of 5D (Fig. 2).

Each stacking sequence was tested to total failure with three specimens for the "Plain", "Small" and "Medium" sizes, and two for the "Large" ones. A third coupon of the "Large" size has been tested up to various percentages of the total failure load as summarized in Table 2.

\subsection{Results}

The test results showed little discrepancy (except for the "Small"/C3-3 combination) for the three coupon sizes combined with the three stacking sequences. Stress/strain curves of coupons $\mathrm{n}^{\circ}$ 3, 6 and 9 of the "Small" specimens (Table 2) are not available because these specimens were filmed edge on, with the initial idea of detecting delamination by means of the infrared camera.

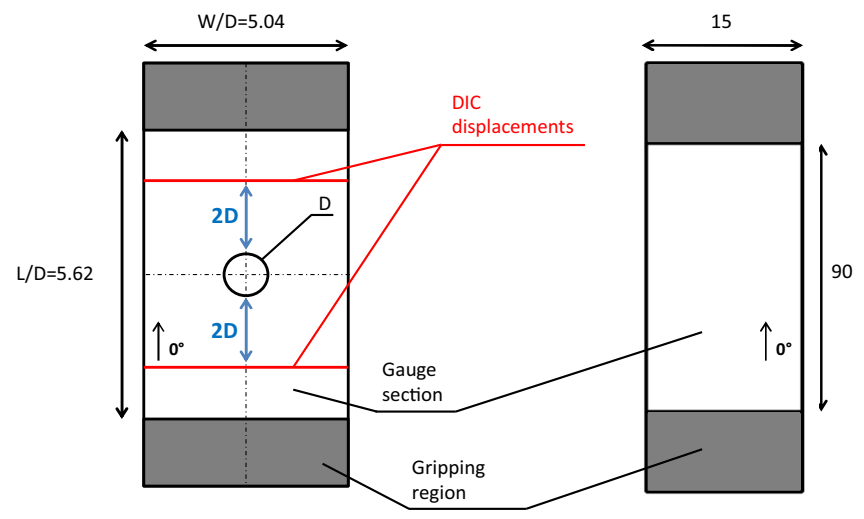

Fig. 2. Open-hole and plain specimen geometries (dimensions in $\mathrm{mm}$ ).
Table 1

Dimensions of the plain and notched specimens ( $\mathrm{mm})$.

\begin{tabular}{llccl}
\hline Specimens & Hole diameter & Gauge width & Gauge length & End tab length \\
\hline "Plain" & N.A. & 15.00 & 90.00 & 50.00 \\
"Small" & 1.00 & 5.04 & 28.35 & 50.00 \\
"Medium" & 3.175 & 16.00 & 90.00 & 50.00 \\
"Large" & 6.35 & 32.00 & 180.00 & 50.00 \\
\hline
\end{tabular}

Delamination damage being energetically very low and also very localized, it was not possible to record useful information about these phenomena. The only valid information was the failure strength reported in Table 3. Oriented Laminate C3-1 was the only layup for which damage recorded by the infrared camera was determined before total failure.

The experimental values presented in Table 3 clearly show a size effect: an increase in the hole diameter from $1 \mathrm{~mm}$ to $6.35 \mathrm{~mm}$ can result in a maximum decrease of $20 \%$ in strength.

$\bar{\sigma}^{\infty}$ refers to the mean remote failure stress and CV to the coefficient of variation. Stress and strain values were normalized by the maximum values encountered in open-hole tests. Maximum stress was reached by coupon $n^{\circ} 9$ (C3-3, "Small"), whose stress/strain curve is not represented.

Consequent variations of plain failure stress were noted (Table 3 ) between composite laminates with the same number of plies in each orientation (only the relative positions differed). These variations were probably due to the relative influence of delamination triggered by free edge effects on each of the stacking sequences studied. Mismatches in Poisson's ratios generated interlaminar stresses [19,39] and hence delamination. The influence of the phenomenon was confirmed by means of experimental observations and a method to predict the onset of delamination in the free edge regions of a symmetric laminate plate subjected to a uniform axial strain, such as the one exposed in the ESDU datasheet [40].

Failure patterns were all of the pull-out type (Fig. 23), which demonstrates that some non-catastrophic damage, such as delamination and ply cracking, appeared before total failure, [8].

\subsubsection{Notched specimens}

2.2.1.1. Oriented laminate C3-1. Regarding "Large" specimens, interrupted tests analyzed by micro-tomography at $20 \mu \mathrm{m}$ resolution were used to determine the extent and nature of the damage at 3 different stages of the test. At $\mathrm{M}_{2}$, no damage was detected whereas, at $\mathrm{M}_{3}, 0^{\circ}$ ply fiber fracture, and $90^{\circ}$ and $\pm 45^{\circ}$ ply matrix cracking and delamination between the outer $\pm 45^{\circ}$ plies were observed (see Fig. 21).

Structural failure (B), defined as the first occurrence of load or stiffness dropping by more than $5 \%$, was probably due to fiber fracture and matrix cracking damage progression observed later on in the test at $\mathrm{M}_{2}$. Fig. 5 shows infrared images from which pictures taken at the beginning of the test have been subtracted. The temperature scale is therefore in variation of ${ }^{\circ} \mathrm{C}$. Red and black areas around the notch, which indicate cooling and warming, respectively, are simply artifacts due to the presence of the hole and the displacement of the coupon. They should not be taken into account in the interpretation of results. At A, the heat emitted by damage propagation was observed with the infrared camera, confirming that fiber fracture occurred and propagated until total failure of the specimen. Even though the shape of heat emission at A, near the hole (Fig. 5), was oriented towards the $\pm 45^{\circ}$ direction, the temperature rise was assumed to come from fiber fracture in $0^{\circ}$ plies. Indeed, heat propagation speed was largest in the direction of the fibers and upper plies were oriented at $-45^{\circ}$ and $45^{\circ}$. 


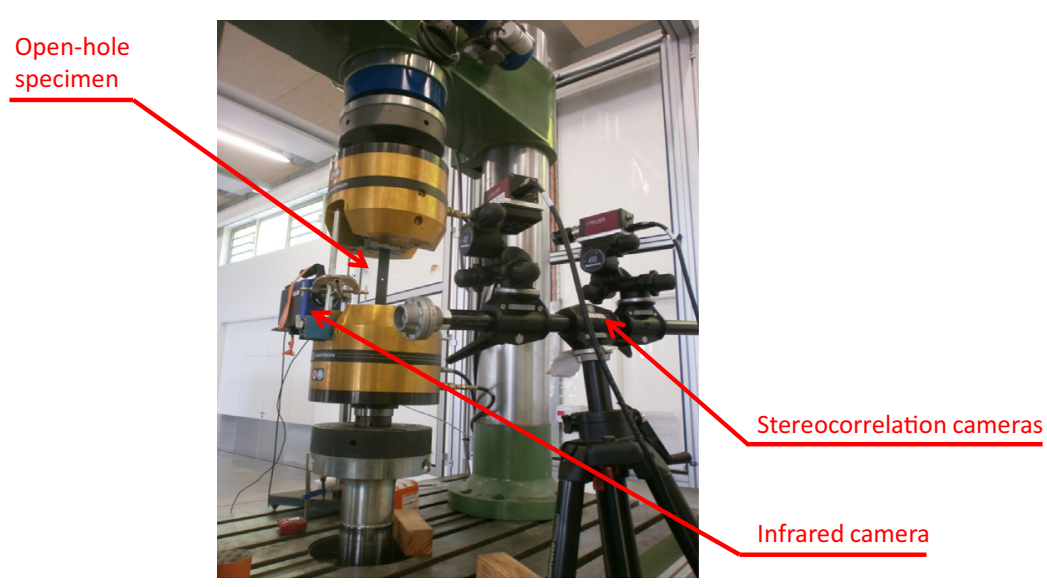

Fig. 3. Experimental setup for the "Large" open hole quasi static tension test.

Table 2

Test matrix.

\begin{tabular}{lll}
\hline Specimens & $\begin{array}{l}\text { Coupons tested until failure } \\
\text { numbering }\end{array}$ & $\begin{array}{l}\text { Interrupted tests coupons } \\
\text { numbering }\end{array}$ \\
\hline "Plain" & C3-1 (1,2,3) & N.A. \\
"Small" & C3-2 (4,5,6) & \\
"Medium" & C3-3 (7,8,9) & \\
"Large" & C3-1 (1,2), C3-2 (4,5), C3-3 (7,8) & C3-1 (3), C3-2 (6), C3-3 (9) \\
\hline
\end{tabular}

Table 3

Results of open-hole tensile tests.

\begin{tabular}{|c|c|c|c|c|c|c|}
\hline \multirow[t]{2}{*}{ Specimens } & \multicolumn{2}{|l|}{ C3-1 } & \multicolumn{2}{|l|}{ C3-2 } & \multicolumn{2}{|l|}{ C $3-3$} \\
\hline & $\bar{\sigma}^{\infty}(\%)$ & CV (\%) & $\bar{\sigma}^{\infty}(\%)$ & CV (\%) & $\bar{\sigma}^{\infty}(\%)$ & CV (\%) \\
\hline “Plain” & 101.1 & 2.5 & 97.8 & 3.7 & 122.7 & 1.5 \\
\hline “Small” & 75.8 & 4.4 & 72.1 & 1.6 & 88.4 & 9.4 \\
\hline “Medium” & 69.6 & 1.7 & 66.8 & 4.5 & 82.8 & 0.7 \\
\hline “Large” & 65.4 & 0.5 & 61.8 & 1.3 & 70.5 & 4.1 \\
\hline
\end{tabular}

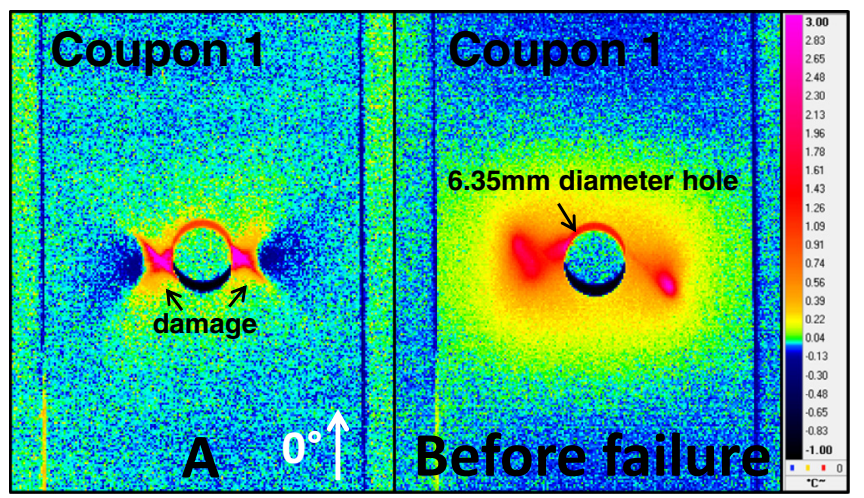

Fig. 5. Damage progression in coupon 1 ("Large", C3-1) observed by infrared camera.

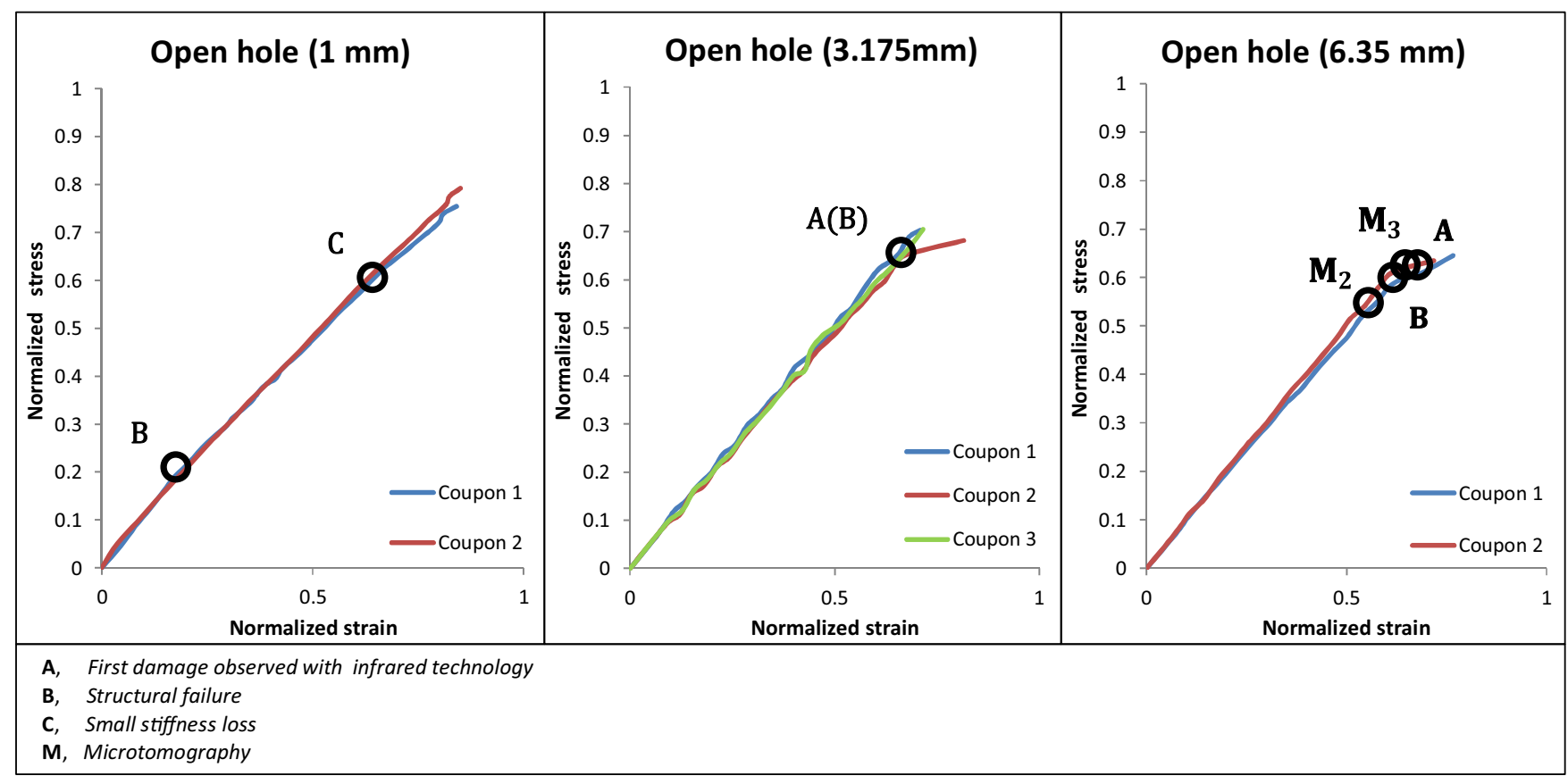

Fig. 4. Experimental hole size effect observed on stacking sequence C3-1. 
Regarding "Medium" specimens, except for coupon 2 where damage appeared before total failure (A-B), the other coupons exhibited a perfectly linear stress/strain curve (Fig. 4) up to complete laminate failure. "Small" specimens had very nonlinear behavior, with two strong stiffness decreases (B and C), which could be explained by the fact that the damage zone was much larger relative to the size of the specimen for the "Small" specimens than for the others.

2.2.1.2. Oriented laminates C3-2 and C3-3. Regarding "Large" specimens, tests interrupted at $\mathrm{M}_{3}$ did not reveal any form of damage. Behavior similar to that of the oriented stacking sequences C3-1 was observed, as represented in Figs. 6 and 7.

\section{Numerical modeling}

The Discrete Ply Model has already been described in several papers [21-25] and the same characteristics were kept here as in Achard et al. for open-hole tension test modeling [38]. The main features, such as mesh construction and behavior law, are briefly recalled below.

The model developed by Bouvet et al. takes account of the three major failure modes in composites: intra-ply fiber failure, intra-ply matrix cracking and inter-ply delamination. As represented in Fig. 8, the mesh consists of 3D volume elements (C3D8) used to model fiber fracture, separated by zero-thickness cohesive elements (COH3D8) to represent delamination and matrix cracking.

The innovative concept here was to have four nodes at each point as shown in Fig. 9. Two of them were used to model matrix cracking of the upper ply and the other two to model the matrix cracking of the lower ply. The two pairs of nodes were connected by delamination elements. Thus, matrix cracking/delamination coupling was achieved naturally. It should be noted that it was necessary to use parallelogram-shaped elements for $\pm 45^{\circ}$ plies in order to make all the nodes coincide.

The in-house hole meshing technique described by Achard et al. [38] was used for the three diameters simulated. The smaller the diameter was, the higher was the number of high-aspect-ratio triangular elements created near the hole edge because of the grid scheme discretization. These elements, often excessively distorted, would lead to numerical divergence and were consequently removed. The shape of the holes, especially the "Small" one (Fig. 30), was not very smooth but there was no contradiction with the physics because drilling generates irregularities. For each simulation, the element size was kept constant at $0.250 \mathrm{~mm}$, i.e. twice the thickness of a single ply. This choice was justified by experimental tests which showed that, within a ply, two matrix cracks tended to be at least 1.5-2 times the ply thickness apart (Fig. 10). Hence, refining the mesh would not be in accordance with the physical reality, and using a rougher mesh (increasing the distance between two matrix cracks) would lead to more numerically unstable, high-aspect-ratio volume elements. Li and Chen [41] determined the minimum distance between two matrix cracks numerically for HS300/ET223 and T300/NY9200Z composite laminates. The results showed a factor of 1.53 and 1.60 between the minimum distance separating transverse cracks and the ply thickness, which confirmed the experimental observations. Saturation crack distance was substantially more affected by the thickness of the cracked ply than by the stiffness of neighboring plies. The influence of mesh size will be thoroughly discussed in Part 4 .

\subsection{Modeling of matrix cracking}

The matrix cracking concept is shown diagrammatically in Fig. 11. To avoid stress concentration, the failure criterion was not calculated within the interface element itself but in the volume elements neighboring it. If the Hashin criterion (1) was reached in either of the volume elements (E1 or E2), then the stiffness of the interface (I1) (initially $10^{6} \mathrm{MPa} / \mathrm{mm}$ ) was set to zero.

$\left(\frac{\left\langle\sigma_{t}\right\rangle^{+}}{Y^{T}}\right)^{2}+\frac{\tau_{l t}^{2}+\tau_{t z}^{2}}{\left(S^{L}\right)^{2}} \leqslant 1$

where $\sigma_{t}$ is the transverse stress, $\tau_{l t}$ and $\tau_{t z}$ are the shear stresses in the (lt) and (tz) planes, \langle\rangle$^{+}$is the positive value, $Y^{T}$ is the transverse failure stress and $S^{L}$ is the shear failure stress of the ply.

When interface elements were used to simulate matrix cracking, diffuse damage was not modeled as only through-the-ply cracks are taken into account in this method. The supporting

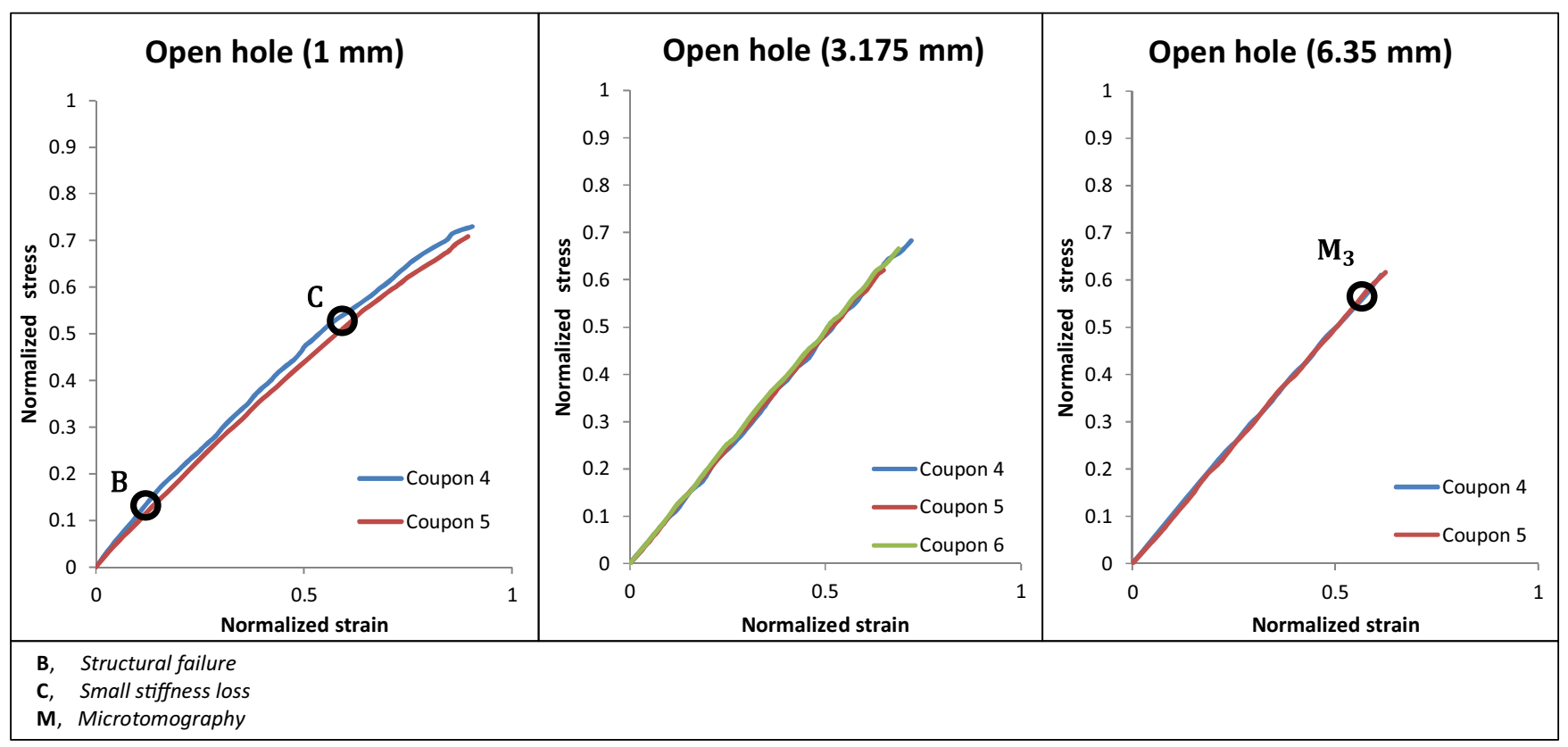

Fig. 6. Experimental hole size effect observed on stacking sequence C3-2. 


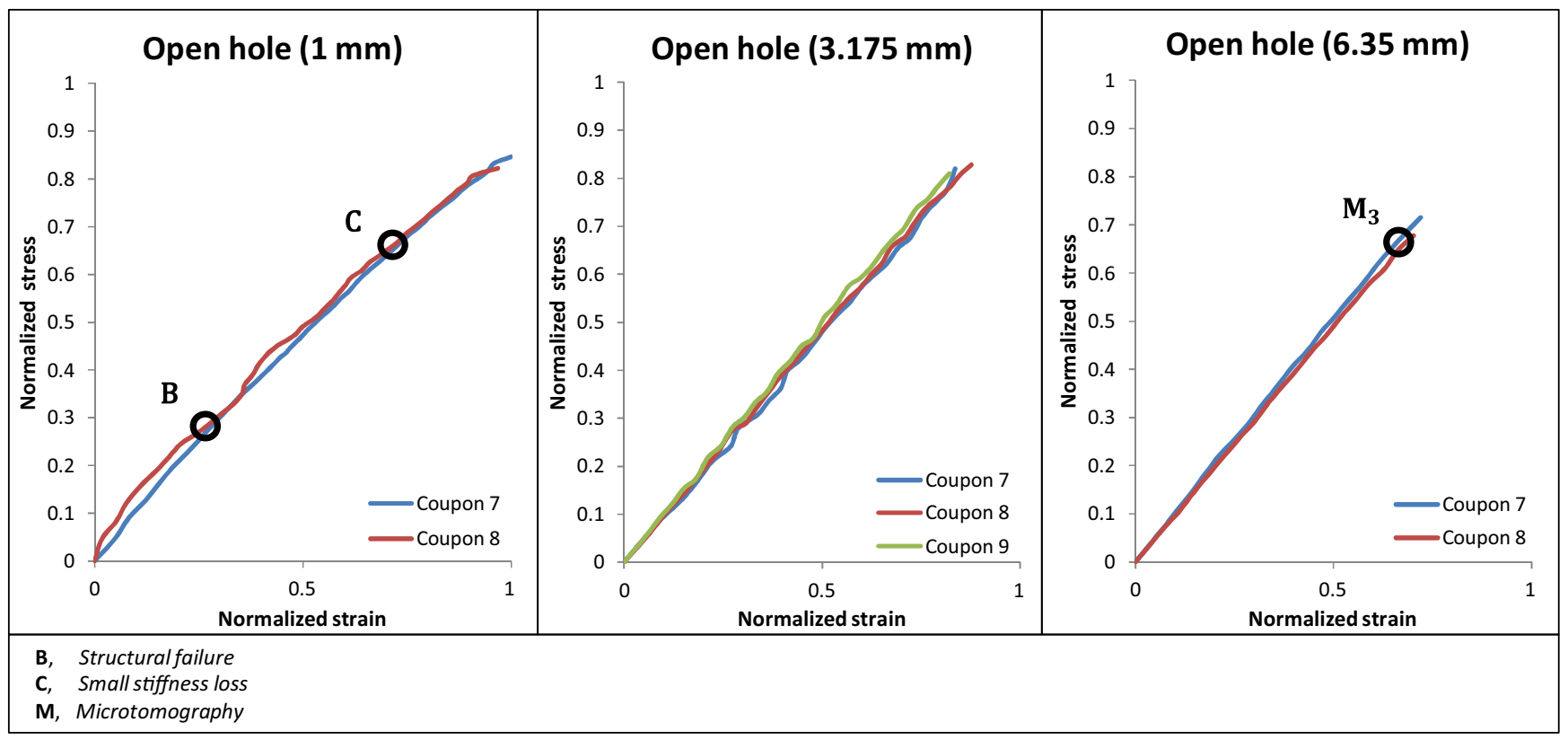

Fig. 7. Experimental hole size effect observed on stacking sequence C3-3.

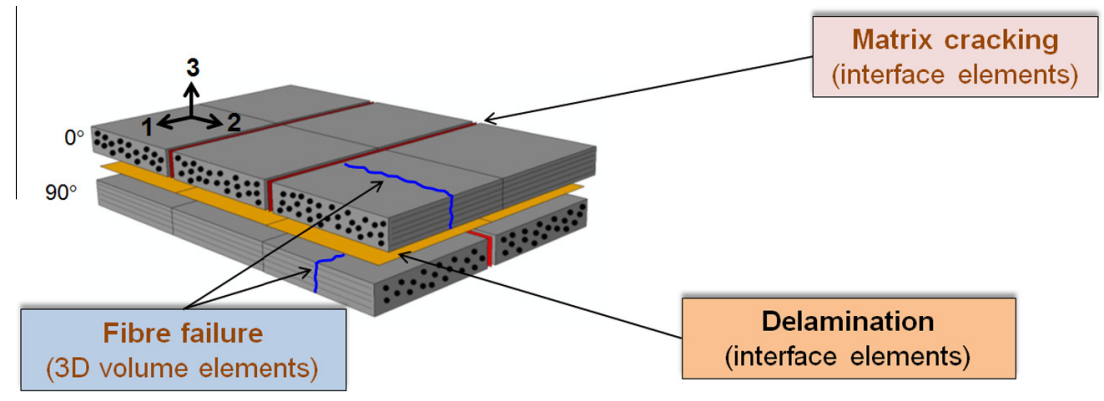

Fig. 8. Discrete Ply Model damage failure modes [21].

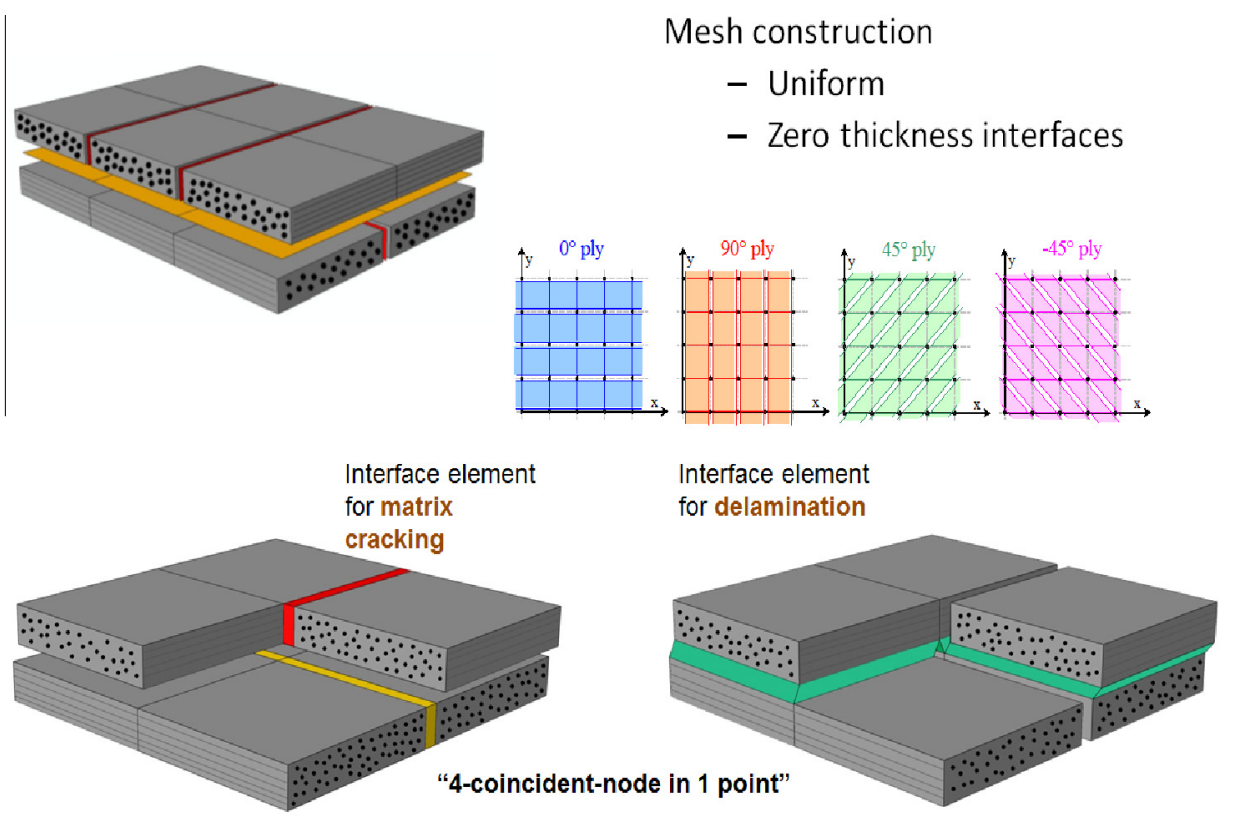

Fig. 9. Mesh specificities of the Discrete Ply Model [21]. 


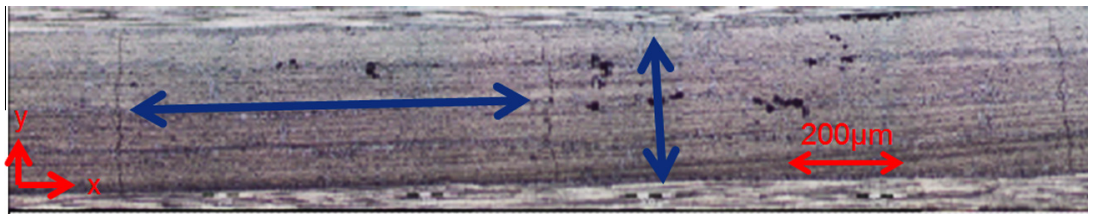

Fig. 10. Matrix cracks within a $90^{\circ}$ ply from a T700/M21 laminate $\left(\left[0 / 90_{4} / 0\right]_{S}\right)$ under three point bending loading (AGI).

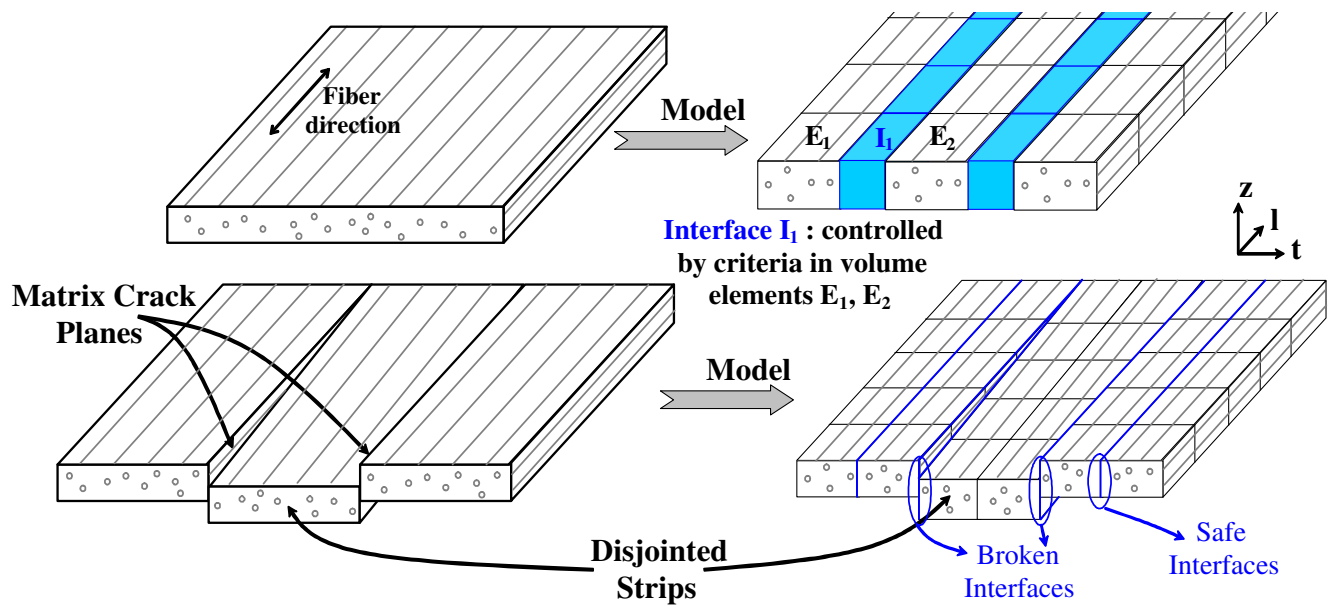

Fig. 11. Discrete Ply Modeling of matrix failure [35].

hypothesis is that a matrix crack propagates fast in a thin ply and therefore diffuse damage should not be represented.

\subsection{Modeling of fiber failure}

As described in Fig. 12, fiber breaking was modeled continuously; the break was initiated when the fiber strain reached the failure strain $\varepsilon_{0}^{T}$. Propagation was included in the model by using the critical energy restitution rate $G_{I c}^{\text {fiber, } t}$. The fiber continued to lengthen until consistent energy had been dissipated (2). The stiffness of the affected volume elements was gradually reduced until fiber strain reached $\varepsilon_{I}^{T}$.

$\int_{V}\left(\int_{0}^{\varepsilon_{I}^{T}} \sigma_{l} \cdot d \varepsilon_{l}\right) \cdot d V=S \cdot G_{I c}^{f i b e r, t}$

where $\sigma_{l}\left(\varepsilon_{l}\right)$ is the longitudinal stress (strain), $V(S)$ is the volume (section) of the element, $\varepsilon_{I}^{T}$ is the strain of total degradation of the fiber stiffness (Fig. 12) and $G_{I c}^{\text {fiber,t }}$ is the energy release rate in opening mode in the direction of the fibers.

First order volume elements were chosen to obtain good bending behavior with only one element in the ply thickness. Two nodes along the thickness direction of the ply is sufficient to describe affine strain variation within the element. A communication law between the 8 integration points was used to ensure that the correct amount of energy was dissipated, on average, per element.

The mesh independency was ascertained by the characteristic length used in the model, which was the volume element length. Constant energy was released per unit area, independently of the element length. This type of approach was first used by Bazant et al. [42] to model concrete failure. It should be noted that other techniques exist for building mesh-independent numerical models. Models based on Cosserat theory [43], non-local models [44], delay effect techniques [45], and combined approaches such as the one developed by Marcin et al. [46] also permit mesh dependency to be reduced by regularization.

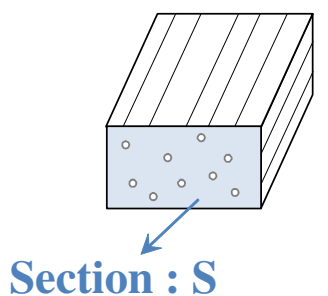

Section : S

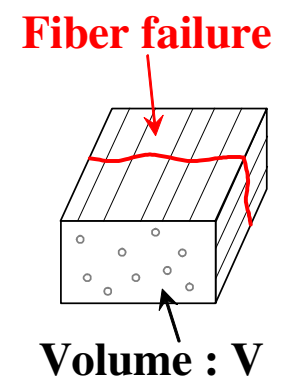

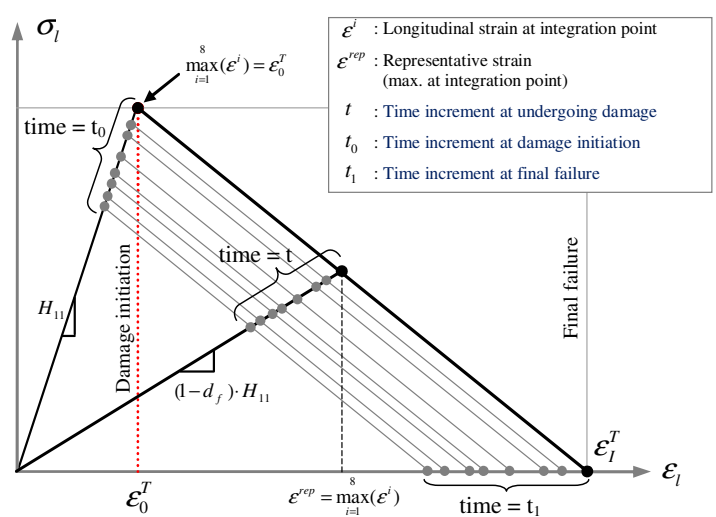

Fig. 12. Discrete Ply Modeling of fiber failure [38]. 


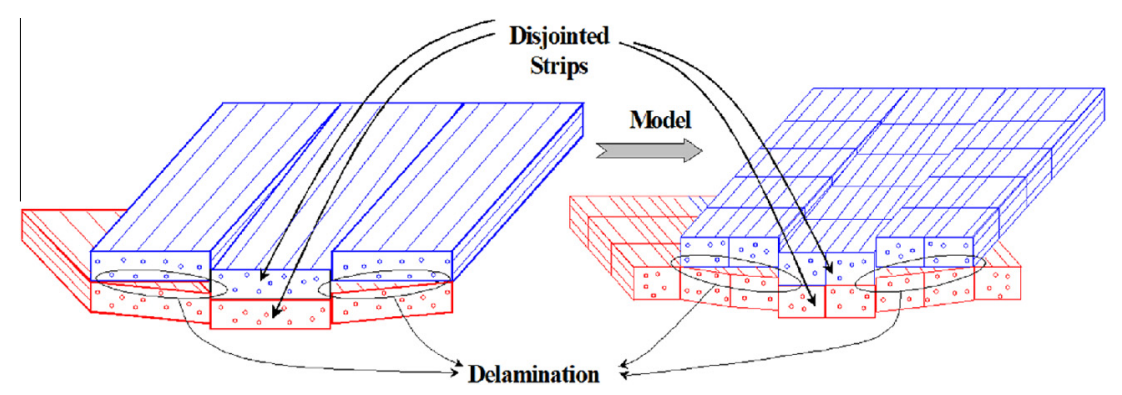

Fig. 13. Discrete Ply Modeling of delamination [35].
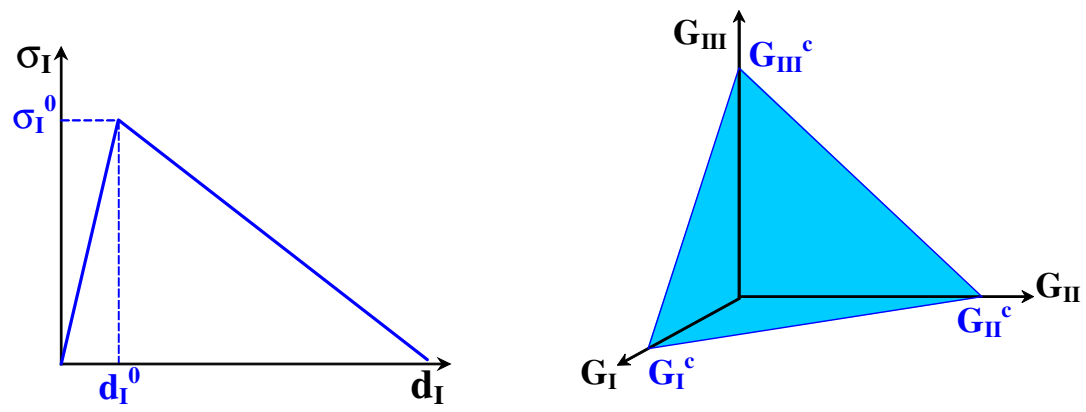

Fig. 14. DPM delamination behavior law [35].

\subsection{Modeling of delamination}

Like matrix cracking, delamination was modeled using cohesive interfaces (Fig. 13). The main difference was that energy was dissipated solely in the case of delamination. Otherwise, the energy dissipated by matrix cracking would be dependent on mesh density.

However, energy spent in matrix cracking was still taken into account since, when the critical energy release rate for delamination was measured, it encompassed delamination energy and most of the energy dissipated through matrix cracking since the DCB test included several types of damage.

Delamination modeling was based on fracture mechanics with a linear softening stress versus displacement behavior law (Fig. 14). According to Hellweg [47], the shape of the unloading curve has a very moderate effect on the overall behavior. For instance, a mode I delamination is initiated when the distance between nodes reaches a critical distance $d_{I}^{0}$, then degradation of the stiffness in mode I is activated and ends when the critical energy release rate is dissipated. To capture the different modes of propagation (I, II and III), equivalent distance and critical energy release rate (Fig. 14) were determined.

\section{Model validation and discussion}

The open-hole tension test was simulated using the finite element model described in Part 3. The mesh was composed of specific elements, detailed in Fig. 9, for the zone near the hole and with linear bricks near the extremities of the gauge zone (Fig. 17). The latter elements, never damaged during the propagation of the crack emanating from the hole, made the calculation faster. Moreover, to further reduce the calculation cost, symmetries were used (Figs. 17 and 18). It was verified that, for the "Medium" and "Large" specimens, modeling one fourth of the coupon (planar symmetry and central symmetry) gave results equivalent to when the whole specimen was meshed. To set planar symmetry corresponding to the laminate symmetry plane, since the three layups studied

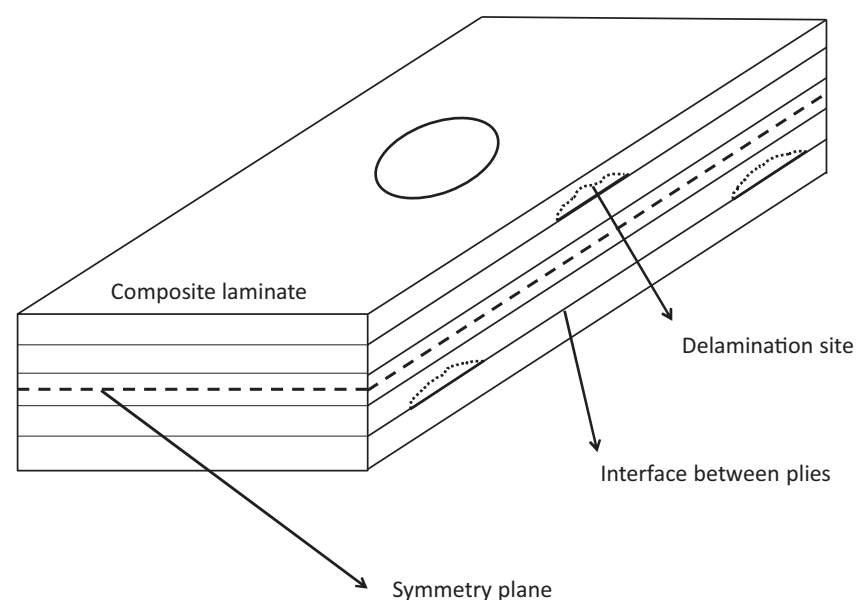

Fig. 15. Schematic diagram of free edge delamination locations on a notched composite laminate.

contained an odd number of plies, the central ply was represented with a thickness of half a ply $(0.0625 \mathrm{~mm})$.

As for the "Small" specimens, an influence of the planar symmetry was observed. The smaller the coupon was, the more substantial was the delamination triggered by free edge effects. This was due to the fact that the smaller the coupon was, the larger was the total failure load and, consequently, since the free edge effects develop for a certain amount of stress, these effects could not reach a sufficient size in the "Medium" and "Large" coupons. By setting planar symmetry, delamination was forced to be symmetric. However, it is likely that, when one interface is partially delaminated its exactly symmetric counterpart does not open because it is being unloaded (Fig. 15). Planar symmetry was consequently removed and one-half of the total mesh was modeled for the "Small" specimens. 


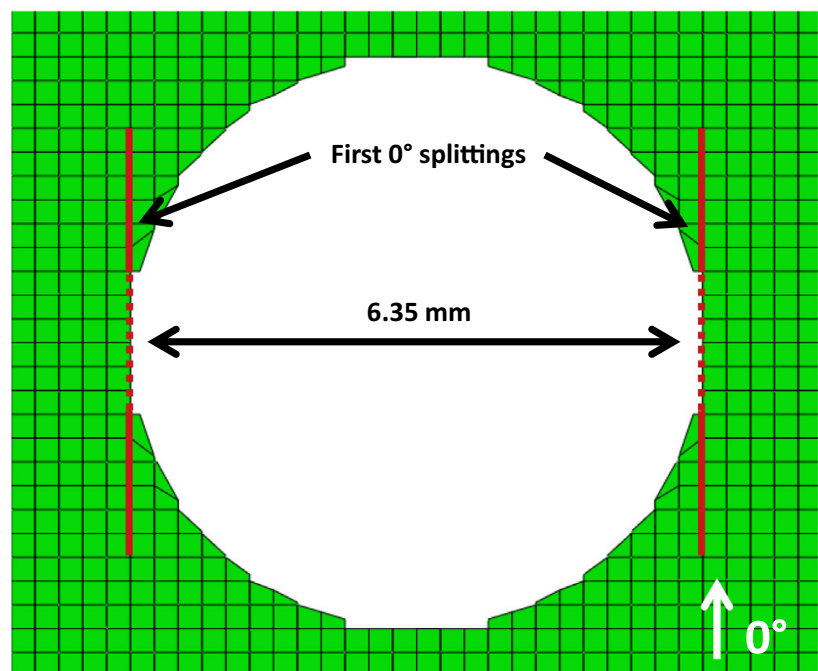

Fig. 16. Location of $0^{\circ}$ matrix cracks in the vicinity of the hole.

Discrete matrix crack elements were inserted within the entire "nonlinear" zone and particular attention was paid to their locations close to the hole. Discrete $0^{\circ}$ matrix crack interface elements were positioned in such a way that the first elements were tangential to the hole on both sides. This allowed splitting to open at the location where the stress concentration was the highest and maximum blunting could thus be achieved, as it was the case in experimental reality. The dotted lines indicate the location where there were no interface elements since neighboring volume elements had been removed because of their high aspect ratio (Fig. 16).

This discrete 3D model was simulated with the Abaqus Explicit solver and a user-defined Vumat subroutine. Pinho's work [48], pointed out issues triggered by this type of solver for quasi-static problems. A damping of the structure is needed to mitigate the dynamic vibration and analysis usually requires a large number of time steps. During these steps, numerical approximations can accumulate, and the work of external forces can eventually be converted into energy other than the internal energy, for example, kinetic energy, hourglass control energy and damping energy. The numerical displacement speed was selected so as to ensure that such additional energy was negligible, while keeping a reasonable calculation time by using multiple CPUs in parallel. A displacement rate of $100 \mathrm{~m} \cdot \mathrm{s}^{-1}$ was used for the "Large" specimens and scaled for the "Medium" and "Small" sizes to keep a constant strain rate. A major advantage of using an explicit finite element code is that it ensures good convergence calculations and, in our case, it overcame the difficulties of making the interface elements converge in implicit codes [48]. Computation time varied between 15 min (8 CPUs) for the "Small" specimens with 60,000 elements

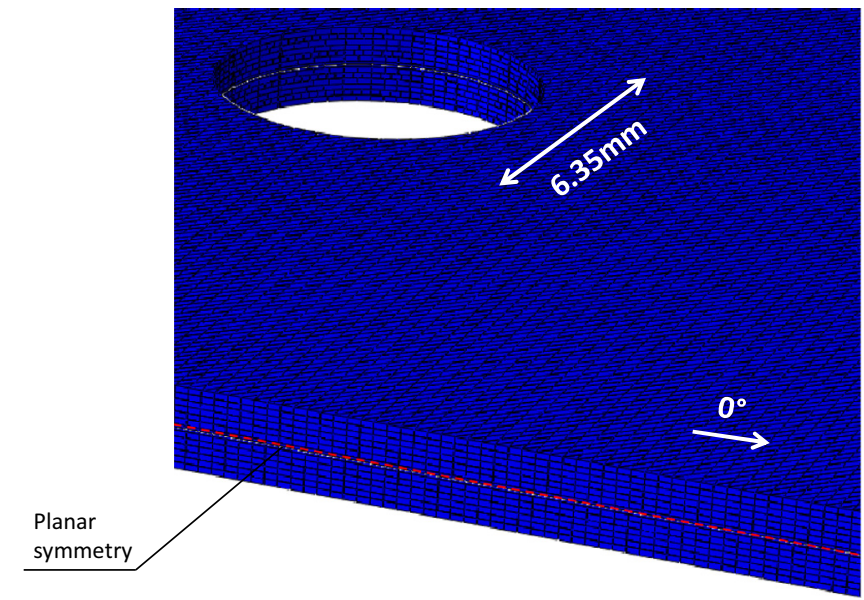

Fig. 18. Close-up view of the $6.35 \mathrm{~mm}$ diameter notched specimen under tension.

Table 4

Material properties of the T700/M21 used in the Discrete Ply Model.

\begin{tabular}{|c|c|c|}
\hline \multicolumn{3}{|c|}{ Material properties } \\
\hline Density & & $1.6 \cdot 10^{3} \mathrm{~kg} / \mathrm{m}^{3}$ \\
\hline \multicolumn{3}{|c|}{ Orthotropic elastic properties } \\
\hline$E_{1}^{T}$ & Tensile Young's modulus in fibre direction & $130 \mathrm{GPa}$ \\
\hline$E_{1}^{C}$ & Compressive Young's modulus in fibre direction & $100 \mathrm{GPa}$ \\
\hline$E_{2}$ & Transverse Young's modulus & $7.7 \mathrm{GPa}$ \\
\hline & Poisson ratio & 0.3 \\
\hline$G_{12}$ & Shear modulus & $5.0 \mathrm{GPa}$ \\
\hline \multicolumn{3}{|c|}{ Matrix cracking } \\
\hline$Y^{T}$ & Transverse tensile strength & $60 \mathrm{MPa}$ \\
\hline$S^{L}$ & In-plane shear strength & $110 \mathrm{MPa}$ \\
\hline \multicolumn{3}{|c|}{ Fiber failure } \\
\hline$\varepsilon_{0}^{T}(\%)$ & Tensile strain in fibre direction at damage initiation & $1.70 \%$ \\
\hline$\varepsilon_{0}^{C}(\%)$ & $\begin{array}{l}\text { Compressive strain in fibre direction at damage } \\
\text { initiation }\end{array}$ & $1.25 \%$ \\
\hline$G_{I c}^{\text {fiber }, t}$ & Fracture toughness for mode I in traction & $100 \mathrm{~N} / \mathrm{mm}$ \\
\hline$G_{I C}^{\text {fiber }, c}$ & Fracture toughness for mode I in compression & $20 \mathrm{~N} / \mathrm{mm}$ \\
\hline \multicolumn{3}{|c|}{ Delamination } \\
\hline$G_{I c}^{d e l}$ & Interface fracture toughness for opening mode (I) & $0.5 \mathrm{~N} / \mathrm{mm}$ \\
\hline$G_{I I, c}^{\text {del }}$ & Interface fracture toughness for shear mode (II \& III) & $1.6 \mathrm{~N} / \mathrm{mm}$ \\
\hline
\end{tabular}

and $50 \mathrm{~h}$ (20 CPUs) for the "Large" specimens with 750,000 elements.

The DPM uses a small number of parameters (Table 4) that are all provided by experimental tests [49], no coupling parameter is required.

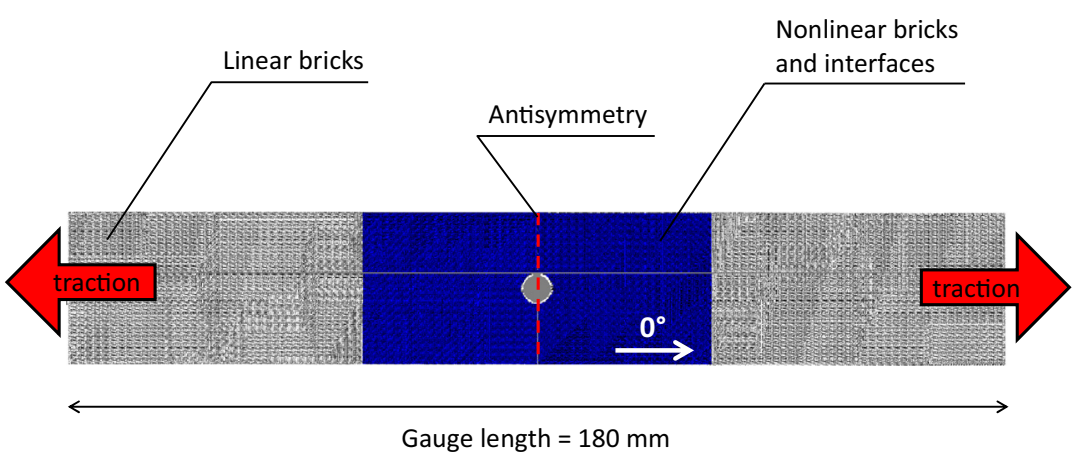

Fig. 17. Numerical model of the $6.35 \mathrm{~mm}$ diameter notched specimen under tension. 


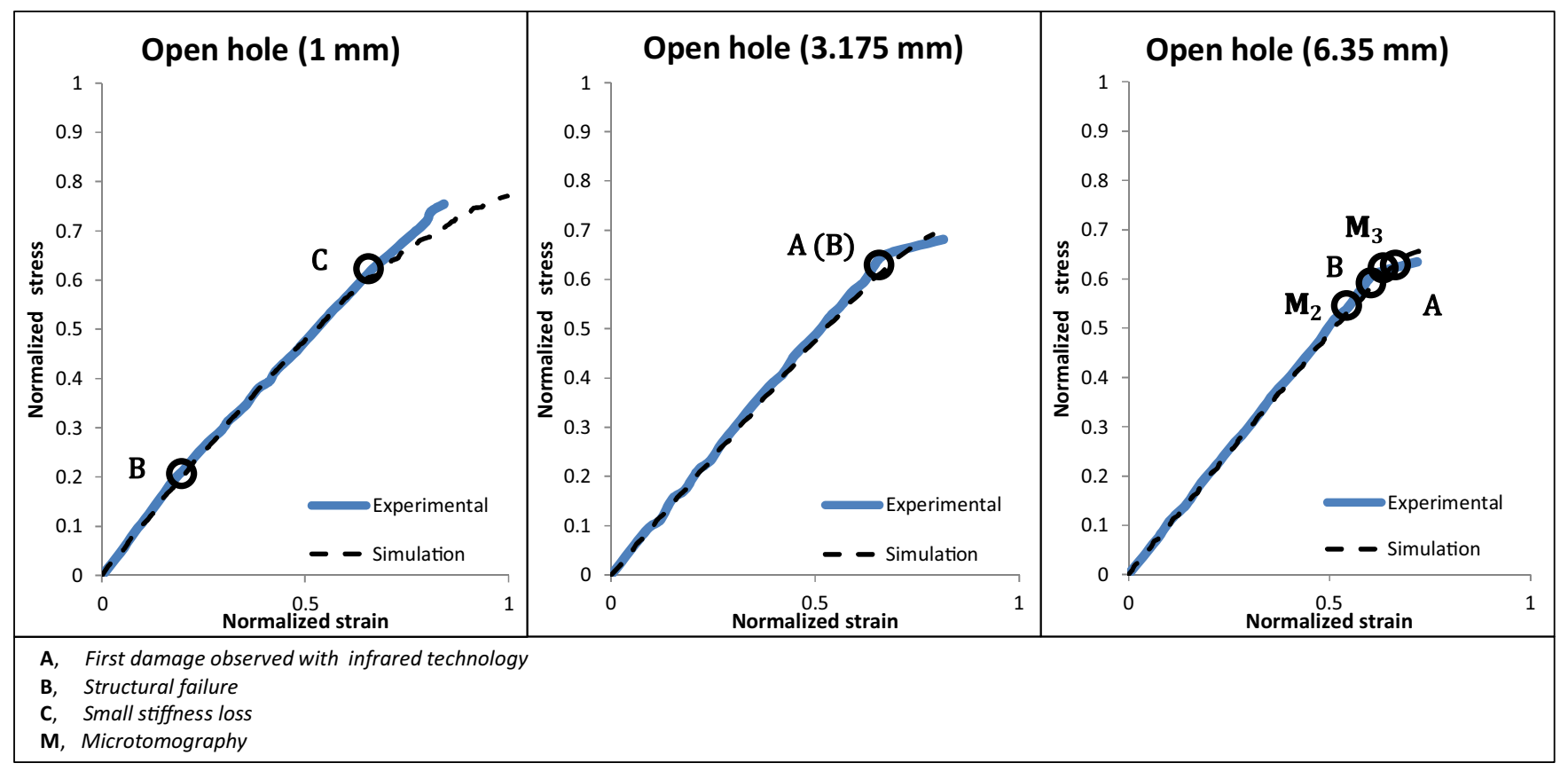

Fig. 19. Stress/strain curves of the three sizes of coupon (C3-1 layup) - comparison between numerical and experimental data.

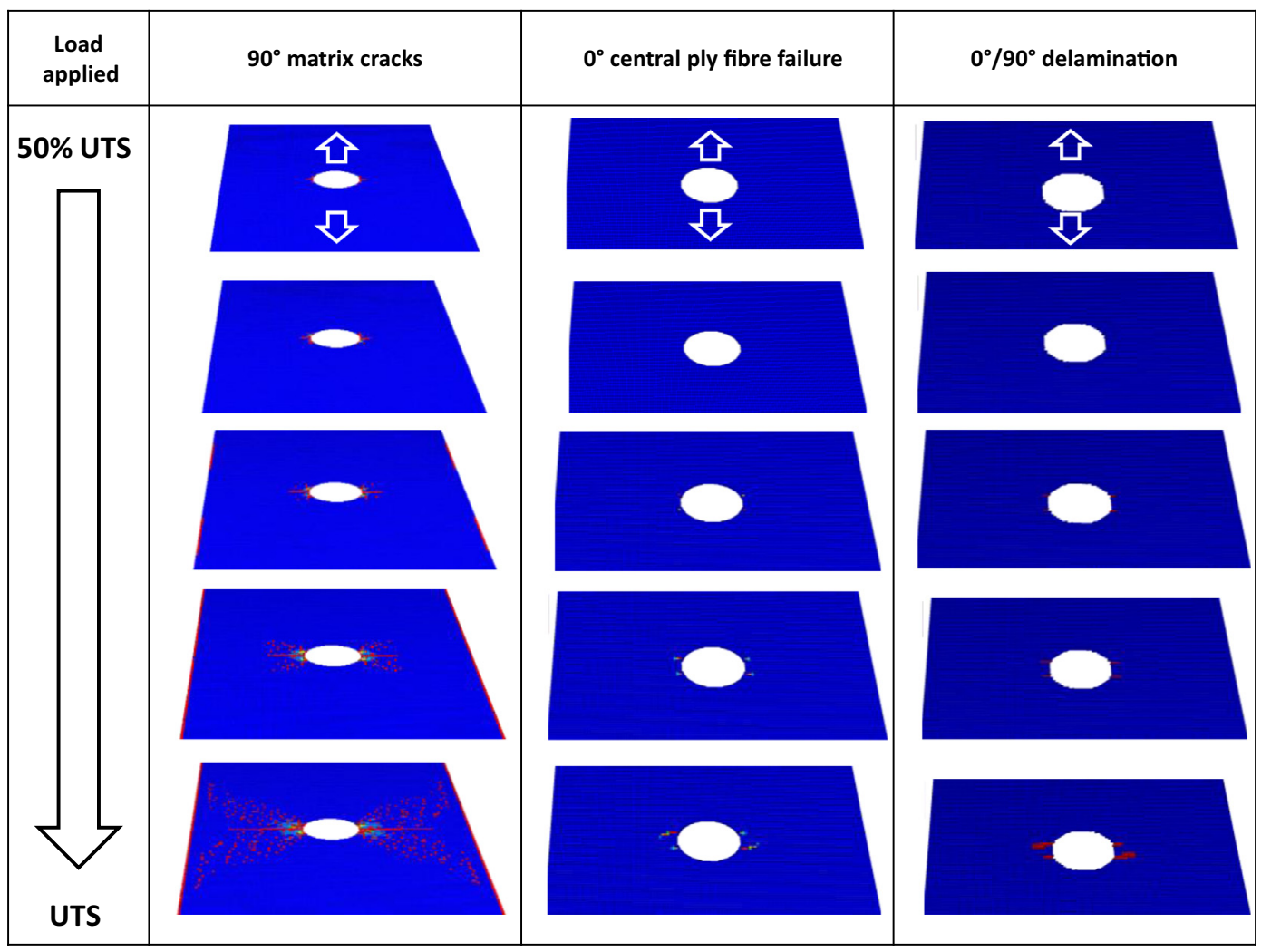

Fig. 20. Matrix cracking in $90^{\circ}$ plies (Column 1), fiber failure of the central $0^{\circ}$ ply (Column 2 ), and $0^{\circ} / 90^{\circ}$ delamination (Column 3 ) of the C $3-1$ "Large" specimen.

\subsection{Oriented laminate C3-1}

Stress/strain curves obtained from numerical simulations are compared with experimental ones in Fig. 19. The elastic part of the tests was very similar in simulation and experiment. It can be seen that the failure strengths are very well predicted for the three sizes.
Structural failure (B) and the other stiffness loss (C) were also determined numerically.

The only difference between experiment and simulation lay in the amplitude of the stiffness loss (C) for the "Small" specimen, which was slightly exaggerated by the model. This was probably due to the extent of the damage being too great compared with experiments. 
Infrared emissions throughout testing and micro-tomography on interrupted tests helped validate the model through comparisons of failure scenarios and patterns.

\subsection{1. "Large" specimens}

The failure scenario of the $6.35 \mathrm{~mm}$ diameter notched C3-1 laminate obtained numerically is shown in Fig. 20 for a $90^{\circ}$ ply (matrix cracks), the $0^{\circ}$ central ply (fiber failure) and for one $0^{\circ} / 90^{\circ}$ interface.

$90^{\circ}$ matrix cracking appeared near the hole edge early in the traction test and propagated a little in the $90^{\circ}$ direction, then cracks appeared at free edges while damage propagated rapidly from the hole edge in a butterfly wing pattern, already observed by Achard et al. in [38].

$0^{\circ}$ fiber failure appeared a short time before ultimate tensile failure and generated sufficient stiffness loss to create a structural failure (B). Delamination $\left(0^{\circ} / 90^{\circ}\right)$ was observed as a consequence of the fiber failure and was very closely confined to the hole vicinity.
To validate the results obtained numerically, the extent of different types of damage at $\mathrm{M}_{2}$ and $\mathrm{M}_{3}$ were compared to damage observed by micro-tomography.

At $\mathrm{M}_{2}$, simulation confirmed the absence of non-critical damage revealed by micro-tomography. At $\mathrm{M}_{3}$, simulation predicted the extents and locations of the various damage occurrences reasonably well (Fig. 21).

Another means to compare numerical and experimental results is through the extent of fiber failure before brutal propagation. Lisle et al. proved that infrared emissions gave accurate estimations of damage growth in thin woven composite laminates subjected to tensile loading [50]. This method was adapted to notched laminated composites under tension. Moreover, fiber failure extent was also compared with micro-tomography output for confirmation (Fig. 22).

The infrared emission showed a crack extension of $20 \%$ of the remaining ligament (two diameters long) while the microtomography revealed values of only $18.5 \%$ (left crack) and $19 \%$ (right crack).

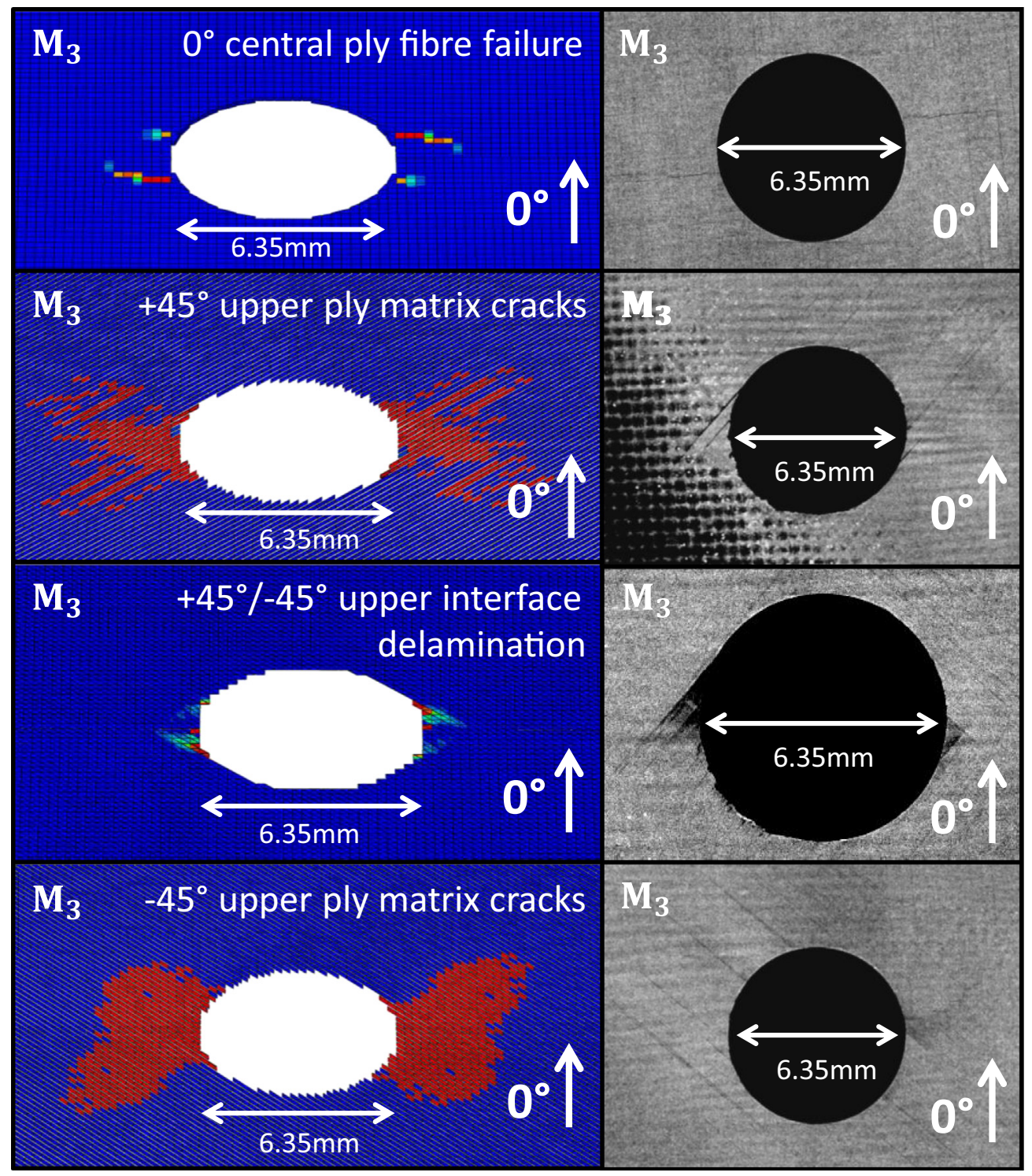

Fig. 21. Comparison between micro-tomography and simulation results at $M_{3}$. 


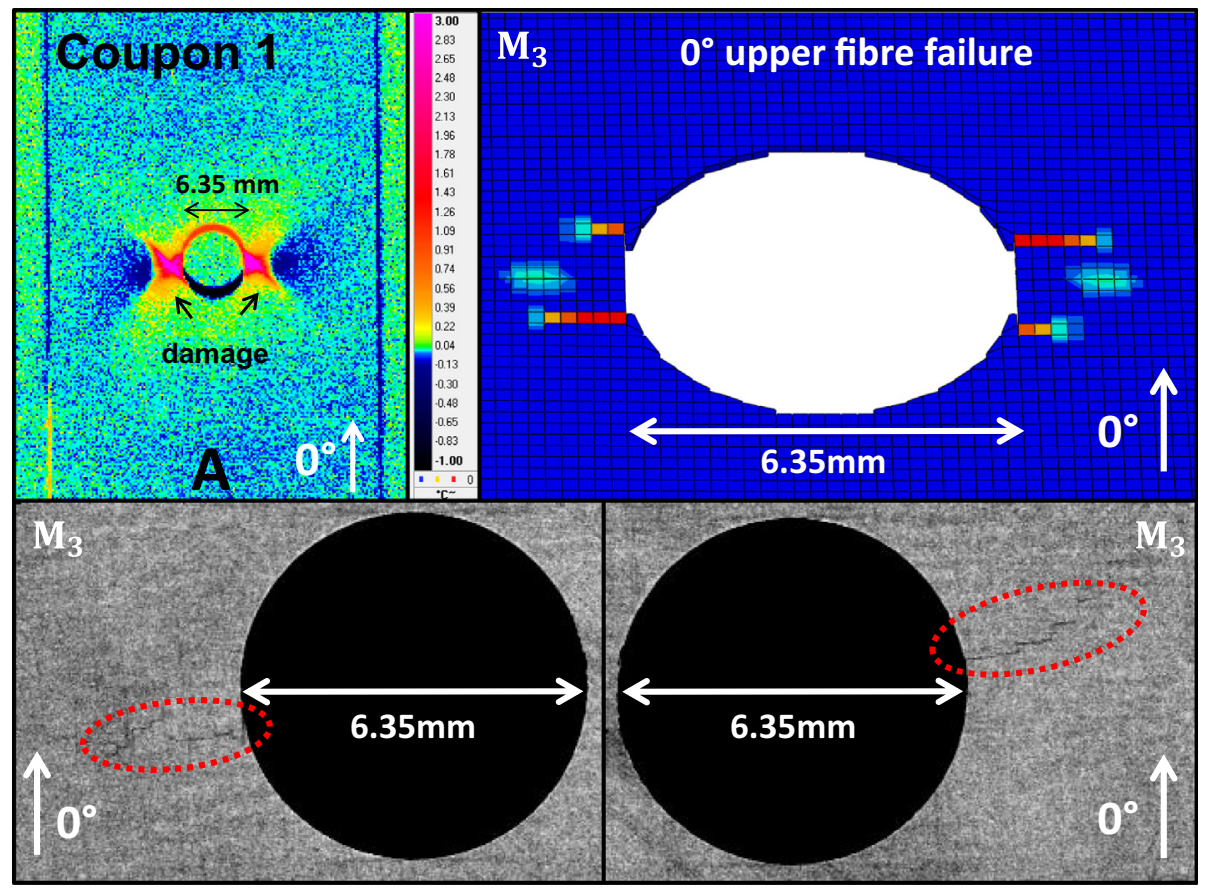

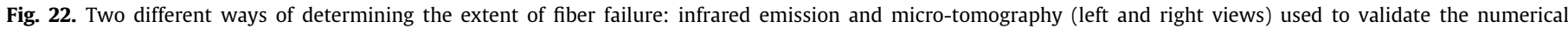
simulation.

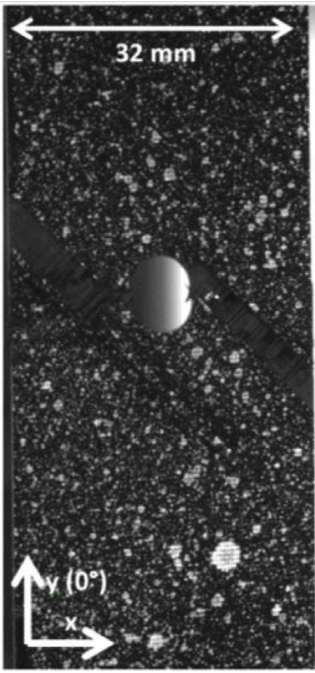

a) b)

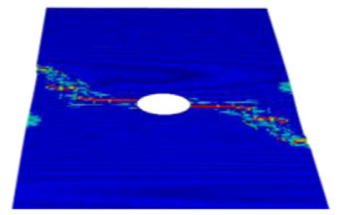

c)

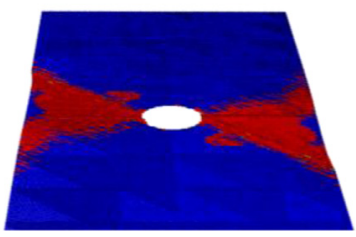

d)

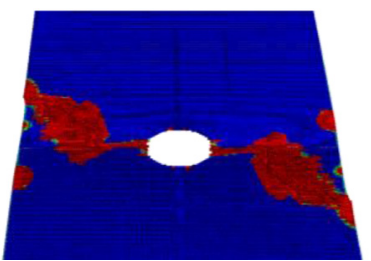

Fig. 23. Comparison of failure pattern for C3-1 "Large" specimen - (a) experimental, (b) fiber failure in outer $0^{\circ}$ ply, (c) matrix cracking in outer $45^{\circ}$ ply and (d) delamination in $0^{\circ} / 90^{\circ}$ interface.

Numerically, the crack extended to $17 \%$, which is quite consistent with experimental observations.

The final failure pattern (Fig. 23) obtained numerically was very similar to the one obtained experimentally. The type (pull-out) and orientation were correctly simulated.

The different types of damage propagated progressively in a direction perpendicular to the tensile direction and, when brutal failure occurred, propagated along the $+45^{\circ}$ direction.

"Medium" specimens did not behave very differently from the "Large" ones, so attention was hence focused on the "Small" specimens.

\subsection{2. "Small" specimens}

"Small" specimens seemed to behave similarly to the "Large" ones but with further non catastrophic damage before ultimate total failure (Fig. 24). This explains the higher strength of the $1 \mathrm{~mm}$ diameter notched specimen: damage located in the vicinity of the hole blunts the stress concentration more and the coupon withstands higher loads.

The failure scenario was similar to the "Large" ones except for the presence of the free edge effect, which was slightly noticeable on the $0^{\circ} / 90^{\circ}$ delamination at UTS but much more noticeable in the $90^{\circ}$ matrix cracks. This phenomenon was also present in the "Large" specimen failure scenario but was less developed proportionally to the width of the specimen. Failure patterns of the same type as the "Large" ones (Fig. 23) were also predicted correctly.

\subsection{Oriented laminate $\mathrm{C} 3-2$}

As for C3-1 laminates, according to stress/strain curves (Fig. 25), numerical predictions agreed well with the results from experiments. The absence of damage near the hole noted in micro-tomography $\left(\mathrm{M}_{3}\right)$ was confirmed by DPM simulations.

The main difference between C3-1 and C3-2 was the free edge effect on the $0^{\circ} / 90^{\circ}$ delamination at UTS, which was much more developed for the "Small"/C3-2 (Fig. 26) than for the "Small"/C3-1 configuration (Fig. 24).

\subsection{Oriented laminate $\mathrm{C} 3-3$}

The main feature of this layup was the presence of a stack of three $0^{\circ}$ plies in the center. It has been demonstrated [51] that ply toughness is substantially dependent on the thickness of the $0^{\circ}$ layers. The increase in toughness measured for specimens with thicker $0^{\circ}$ plies seems to be due to an increase in the number of 


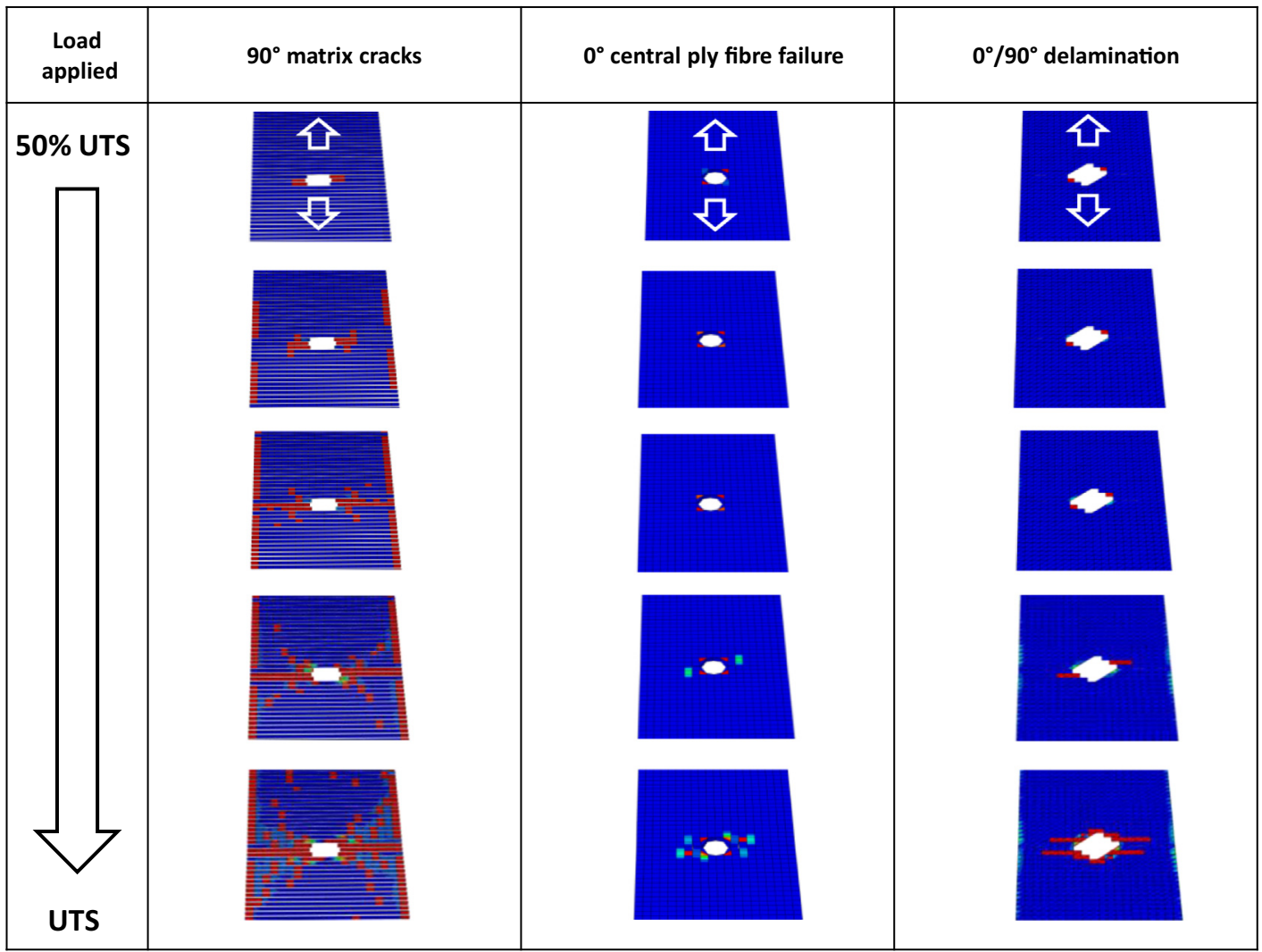

Fig. 24. Matrix cracking in $90^{\circ}$ plies (Column 1), fiber failure of the central $0^{\circ}$ ply (Column 2) and $0^{\circ} / 90^{\circ}$ delamination (Column 3) of the C3-1 "Small" specimen.

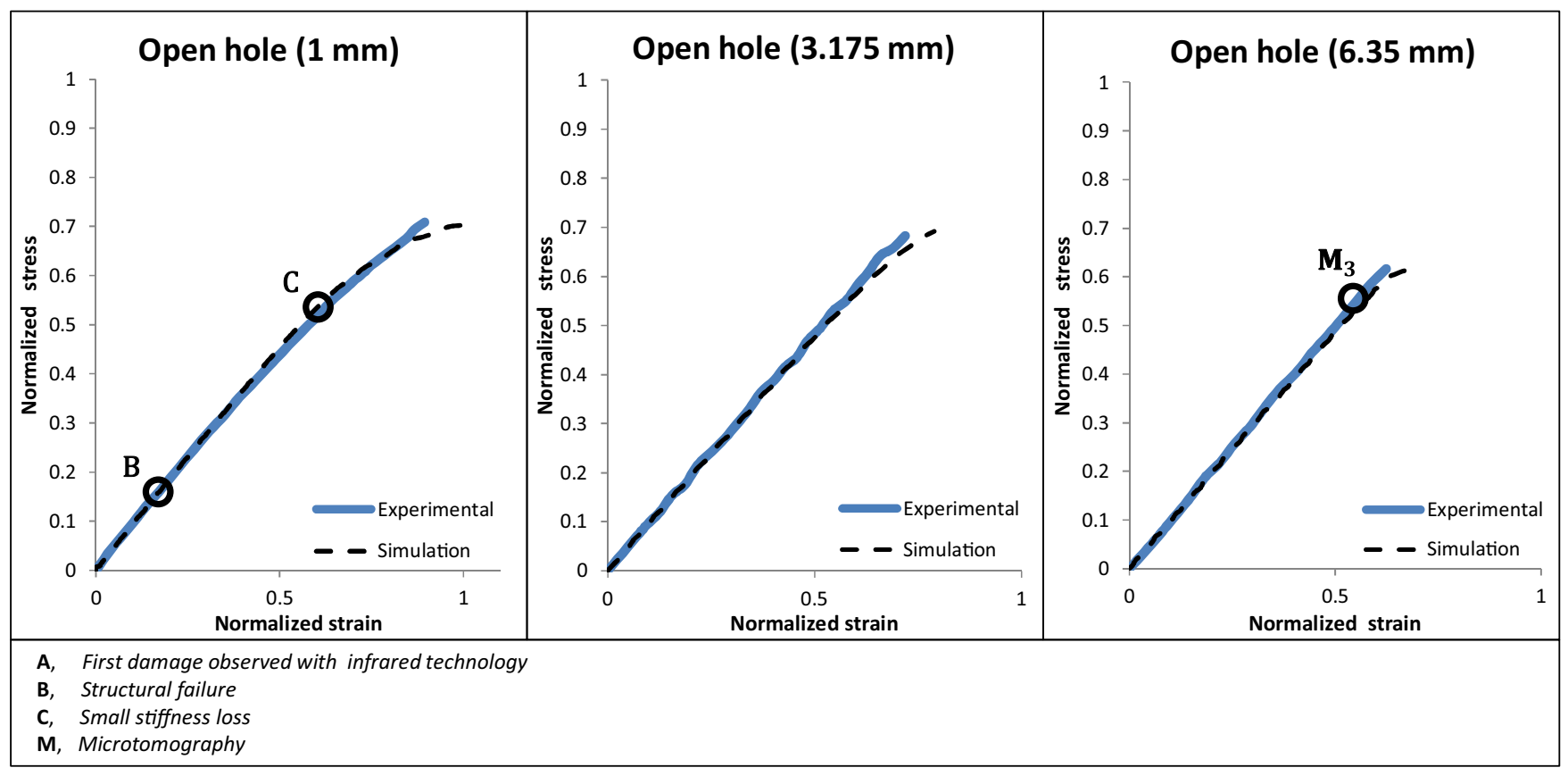

Fig. 25. Stress/strain curves for the three sizes of coupons (C3-2 layup) - comparison between numerical and experimental data.

pulled-out $0^{\circ}$ fibers. Chen et al. were the first to take this dependence into account in the numerical modeling of composites [22] and proved it was necessary to correlate with physical reality. This tendency was confirmed by Ridha et al. the next year on specimens with a large variety of sizes and stacking sequences [25]. Pinho et al. and Ridha et al. used different coefficients to represent the increase in fracture toughness (respectively $k=2$ and 3 ) with $k$ such that:

$G_{I, c}^{\text {stacked plies }}=k \cdot G_{I, c}^{\text {single ply }}$ 


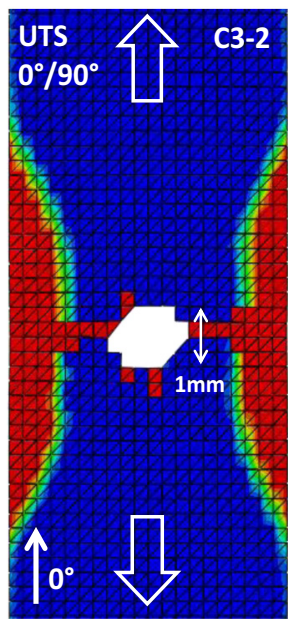

Fig. 26. Numerical simulation of the delamination $0^{\circ} / 90^{\circ}$ for the "Small"/C3-2 combination just before total failure.

Simulations were hence run using both values of fracture toughness for mode I in traction $\left(G_{I, c}^{\text {fiber, } t}\right)$.

For "Small" and "Medium" specimens, both rises in toughness gave more accurate results than the standard case $\left(G_{I, c}^{\text {fiber, }}=100 \mathrm{~N} / \mathrm{mm}\right)$ in terms of failure strength but the modification triggered an overprediction of the failure strain. In addition, when the "Large" specimen was investigated, both failure strain and stress were strongly overpredicted (Fig. 27), even for $k=2$. In consequence, the original configuration was kept.

When very little non catastrophic damage occurs before failure, as was the case for the "Large" specimens, failure is completely driven by fiber failure in the $0^{\circ}$ plies and it was thus to be expected that a multiplication of the fracture toughness by two or three would produce substantial changes in the total behavior of the laminate. In contrast, for "Small" specimens" where much non catastrophic damage occurs, total failure is not solely due to $0^{\circ}$ fiber failure. This is why the change in fracture toughness had less influence in the $1 \mathrm{~mm}$ diameter notched specimen.

\subsection{Discussion}

\subsubsection{Scaling effect}

It has been proven that the DPM correctly transcribes the scaling effect for open-hole tensile tests. Concerning the parameters influencing the simulation results, two main factors have been explored: the presence of matrix cracks and the mesh size on the three layups. Results regarding C3-2 are presented Fig. 29.

When matrix cracks were not inserted, the mesh was either (a) built with elements whose length was twice the thickness of a ply (Ply Sized Elements), or (b) built with element sizes scaled according to the diameter of the hole studied (Hole Sized Elements), i.e. with a constant number of elements along the width of the laminate. The baseline element size used for the "Small" specimens in (b) was thus the same as the one used for the $1 \mathrm{~mm}$ diameter notched specimen in (a). When the matrix cracks were included, the same two types of mesh were tested: (c) and (d).

The difference either between (a) and (b) or between (c) and (d) was small. In both cases, stress/strain curves were very similar except for a slight disparity for the "Large" and "Medium" cases. This disaccord was probably due to the free edge effects (Fig. 15), which were more precisely taken into account in (a) and (c) (several elements on the zone considered) than in (b) and (d) (a single element in the zone considered) as represented in Fig. 28.

According to Eq. (2), the volumetric density of energy dissipated during fiber fracture depends on the length of the volume element. In the specific mesh of the DPM, this length is determined by the mesh density imposed. It then seems that this variation of energy is not responsible for the scaling effect ( $b$ and $d$ ). The distance of the integration points inside the volume elements from the hole edge, which would generate a larger smoothing effect for the largest elements, has no effect either. The mesh does not appear to have much influence on the scaling effect representation and selecting a specific size is not of major importance except when the free edge effects play a consequent role. In that case, opting for a length twice the thickness of a ply gives a reasonable rendering of their influence in the failure scenario.

It should be noted that simulation without matrix cracks (a) and (b) yielded a notched strength higher than the ones with such cracks (c) and (d). This was solely due to the lack of criteria to

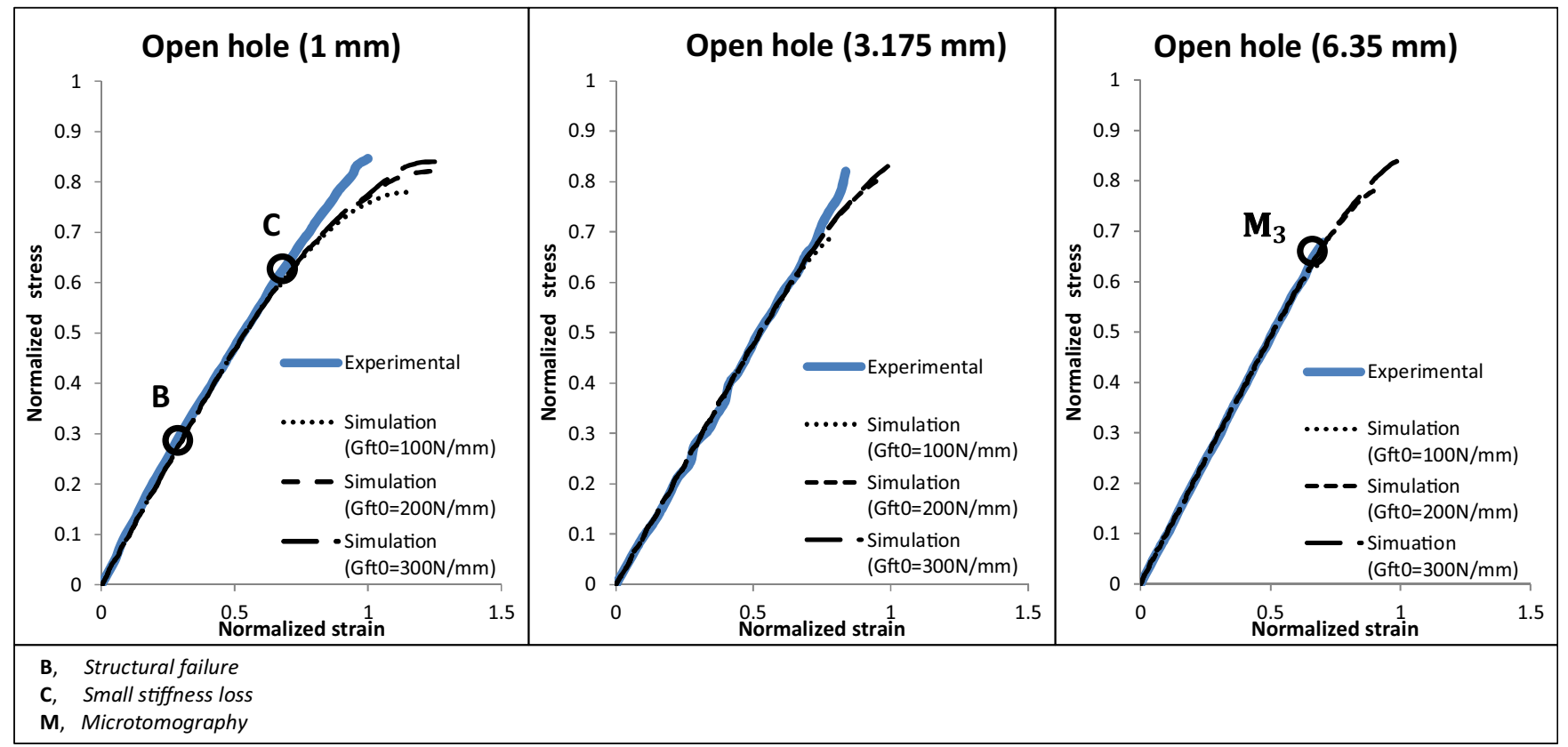

Fig. 27. Stress/strain curves of the three sizes of coupons (C3-3 layup) - comparison between numerical and experimental data. 


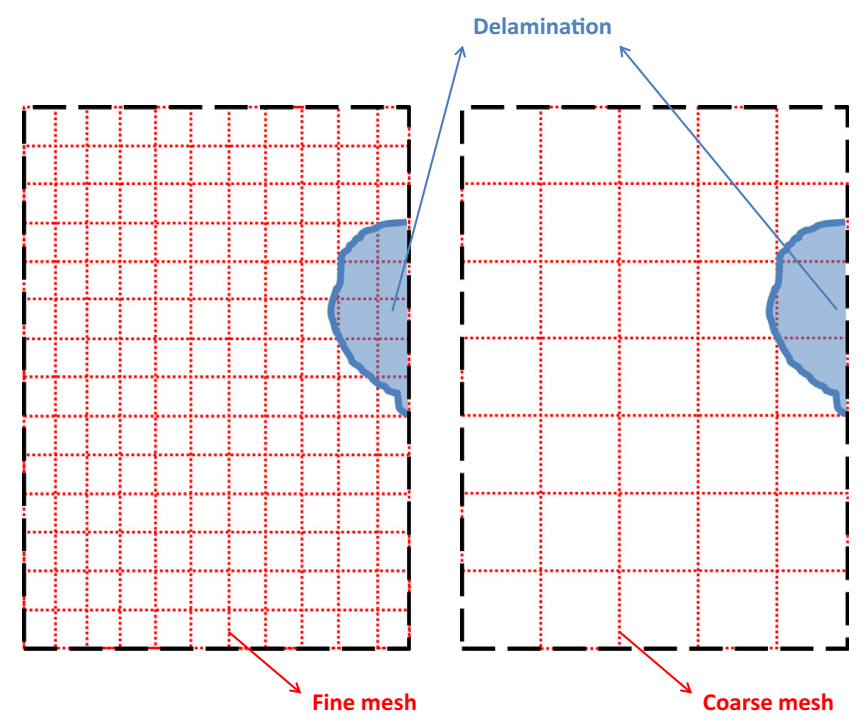

Fig. 28. Delamination discretization with fine and coarse mesh.

replace cohesive interfaces and to describe effect of matrix cracks on laminated composites structural integrity. Only the ratio between the different scales has a meaning, the absolute values alone do not.

When matrix failure is not taken into account, the scaling effect can be over predicted. Between the "Small" and "Large" specimen ranges, failure load increases from $16 \%$ for (d) to $20 \%$ for (b). Compared to the experimentally determined increase (16\%), matrix cracks seem to be indispensable to correctly represent the physics associated with damage progression in an open-hole specimen under tension. Sun et al. reached the same conclusions when modeling quasi-static indentation tests [28]. They used numerical simulations with different levels of discretization, i.e. the number of potential intralaminar cracks varied from no cracks, through single, then double to multiple cracks. The model with multiple cracks predicted the damage scenarios best. This was probably due to the interaction of inter- and intralaminar damage in composites. Matrix cracks seem to initiate delamination and then limit its expansion by enclosing it. Without intralaminar cracks, delamination would be able to develop further and so smooth the stress concentration in larger proportions than experimentally observed (b).

The scaling effect is determined by the relative proportion of non-critical damage in the vicinity of the hole, especially the extents of delamination and fiber failure. Matrix cracks seem to play a role of contention for delamination rather than a directly smoothing role as was the case with the splitting analyzed by Achard et al. using the same model [38]. All the conclusions drawn from the numerical tests were the same for the three layups C3-1, C3-2 and C3-3.

Fig. 30 shows the extent of both delamination and fiber failure for the "Large" and "Small" specimens of layup C3-1. The scaling effect is thus demonstrated.

\subsubsection{Stacking sequence effects}

Stress/strain curves and failure scenarios look globally alike for the three stacking sequences. However, it was observed experimentally that the notched strength of C3-3 was always higher than for C3-2 and C3-1, as noted for the plain specimens. Notch sensitivity varies from one layup to another according to the ratios of net notched strengths to unnotched strengths in Table 5.

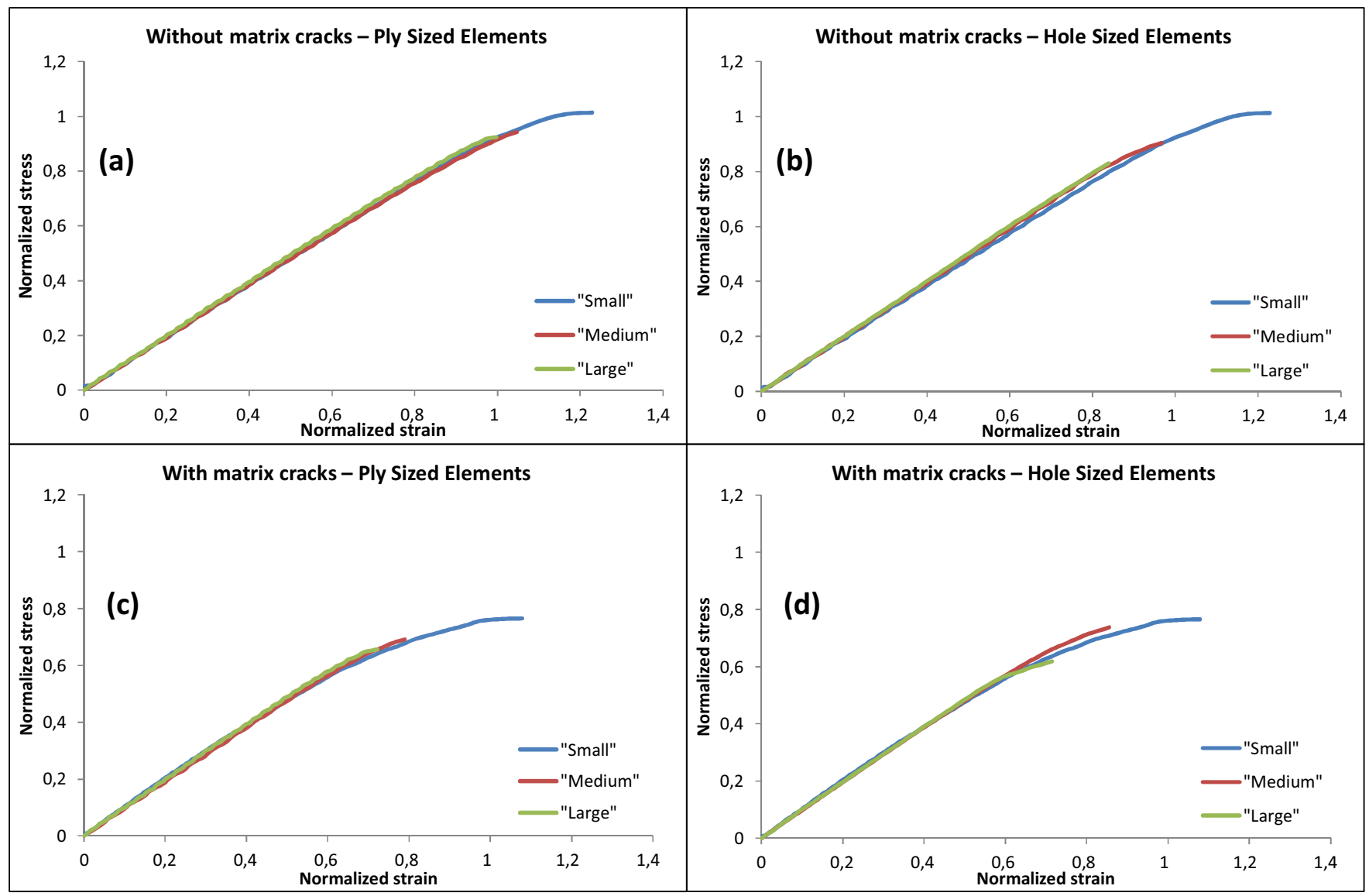

Fig. 29. Influence of discrete matrix cracks and mesh size on stress/strain curves for the three sizes of layup C3-2. 


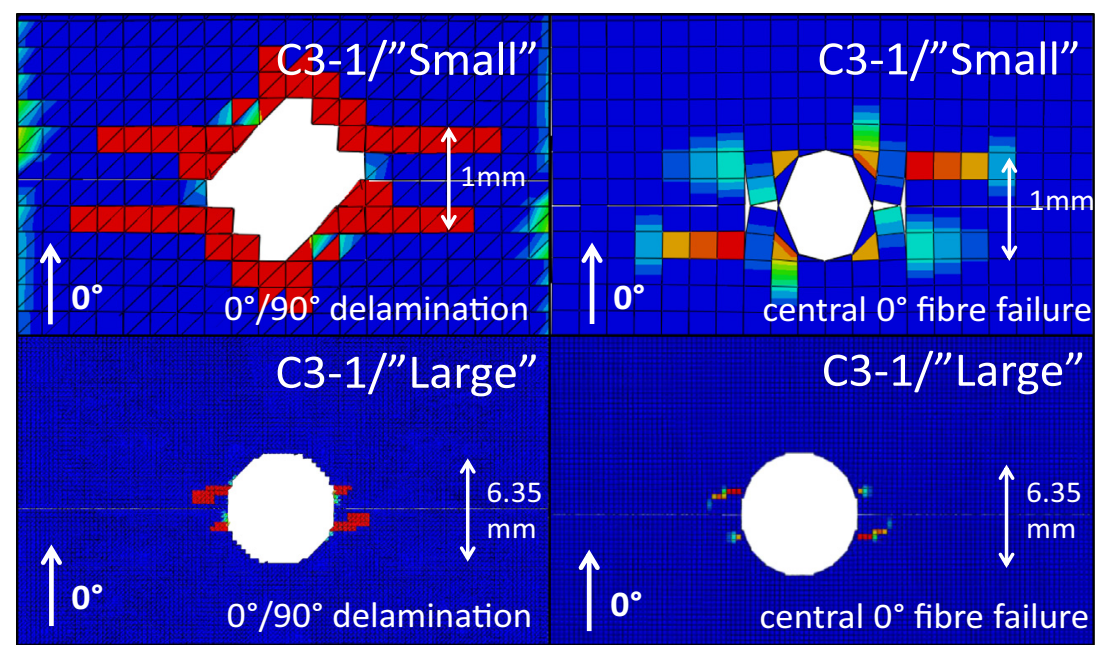

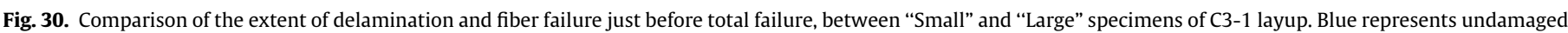

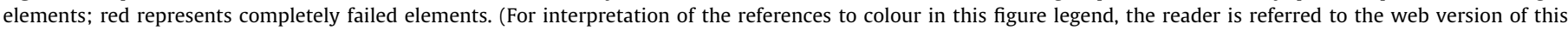
article.)

Table 5

Notch sensitivity of the three layups.

\begin{tabular}{llll}
\hline Specimens & \multicolumn{2}{l}{ net $/ \bar{\sigma}_{\text {unnotched }}$} & \\
\cline { 2 - 4 } & $\mathrm{C} 3-1$ & $\mathrm{C} 3-2$ & $\mathrm{C} 3-3$ \\
\hline "Small" & 0.94 & 0.92 & 0.90 \\
"Medium" & 0.86 & 0.85 & 0.84 \\
"Large" & 0.81 & 0.79 & 0.72 \\
\hline
\end{tabular}

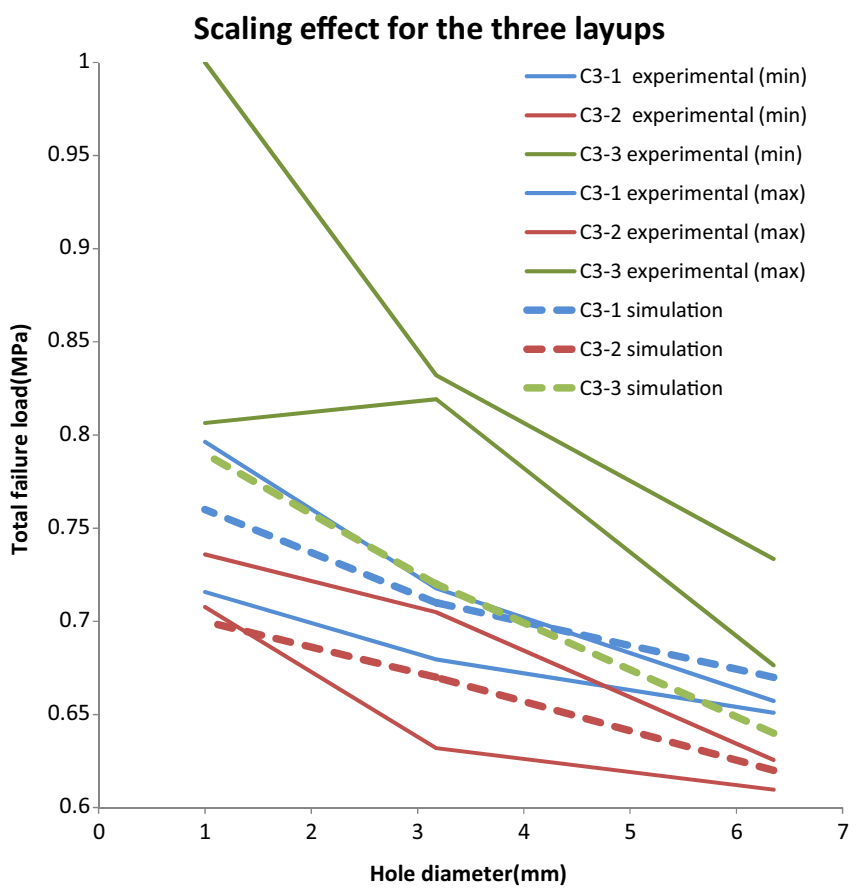

Fig. 31. Scaling effect on the three different layups (C3-1, C3-2 and C3-3).

The smaller the holes were, the less sensitive were the layups, i.e. net notched strength was very close to unnotched strength. This is consistent with the fact that the smaller the hole is, the larger is the blunting stress concentration damage area. "Small" specimens are almost at the point where the stress concentrations triggered by the hole are completely smoothed.
C3-3 layup appears to be the layup that is the most sensitive to the presence of a hole. The ratio $\bar{\sigma}^{\text {net }} / \bar{\sigma}_{\text {unnotched }}$ is lower than for the two other layups. Free edge effects that are preeminent in the C3-1 and C3-2 plain specimens under tension evoked in Section 2.2 seem to have the opposite effect in the case of the open-hole tensile test. Edge effects then occur in the vicinity of the hole, too, and the damage created blunts the stress concentration, which lengthens the life of the composite. C3-3 layup does not smooth stress concentrations as much as the other two layups because of its better resistance to edge effect and to fiber fracture, but it nevertheless has some smoothing effect. Damage accumulation still takes place around the hole and is likely to come from the "clustering effect" [52] i.e. the influence of a group of plies with the same orientation, which results in:

- A thicker ply (group of three plies at $0^{\circ}$ ), which is more prone to matrix cracks that can lead to delaminations.

- An increase in inter-ply shear stresses at the adjacent interfaces $\left(-45 / 0_{3}\right)$ due to the difference in bending stiffness of the individual groups of plies, which in principle enhances the spread of delamination.

- A reduction in the number of interfaces (10 for C3-3), which with the same absorbed energy, may delaminate more than another layup with a greater number of interfaces (12 for C3-1 and (3-2).

Numerical simulations gave excellent results for C3-1 and C3-2 layups (Fig. 31). C3-3 simulations underpredicted the experimental results, probably because of the influence of the fiber failure fracture toughness (Section 4.3), not taken into account in the results presented (Fig. 31). Maximum disparity between numerical and experimental results was $12 \%$ (C3-3, "Medium”).

\section{Conclusion}

Predicted notched tensile strengths of T700/M21 composite laminates decreased with specimen size for the three stacking sequences studied, as observed experimentally. The DPM approach, previously applied to open-hole tension simulation, was directly extended to represent the scaling effect through three diameter sizes here. The three most current damage types were simulated: matrix cracking, fiber failure and delamination. Stress/ strain behaviors, and failure scenarios and patterns were correctly 
described. The importance of the relative positions of plies was shown experimentally, especially through edge and cluster effects. The influence of the layup was then simulated with reasonable accuracy via the DPM.

The influence of mesh size and presence of discrete matrix cracks has been discussed. Mesh size does not seem to have a substantial effect on numerical response except for edge effect transcription and the fact that omitting matrix cracks might lead to oversized delamination extents. Thus it appears necessary to implement discrete matrix cracks in the model and to have a mesh size such that the distance between two cracks is $1.5-2$ times the thickness of the ply considered.

The originality of the model comes from its discrete nature and the absence of coupling parameters. Effort has also been put into modeling " $U$ notch" propagation under tension within the same stacking sequences (C3-1, C3-2 and C3-3) and a comparison with this paper's results will be presented shortly. An extension to validate this approach with composite structures under complex loadings is also in progress [49].

\section{Acknowledgements}

The research that led to the results presented above received funds from the French National Research Agency under the VERTEX project MATETPRO program (ANR - 12 - RMNP-0001). The academic authors gratefully acknowledge the support provided by Airbus Group Innovation. This work was granted access to the HPC resources of CALMIP under allocation 2012-P1026.

\section{References}

[1] Gay D. Matériaux composites. 6e édition revue et augmentée. Paris: Hermes Science Publications; 2015.

[2] Kassapoglou C. Modeling the effect of damage in composite structures: simplified approaches. s.l: Wiley; 2015.

[3] Bažant ZP. The scaling of structural strength. London: HPS; 2002 (1st publ.).

[4] Bažant ZP, Daniel IM, Li Z. Size effect and fracture characteristics of composite laminates. J Eng Mater Technol 1996;118(3):317-24.

[5] Wisnom MR, Hallett SR, Soutis C. Scaling effects in notched composites. ] Compos Mater 2010;44(2):195-210.

[6] Xu X, Wisnom MR, Mahadik Y, Hallett SR. An experimental investigation into size effects in quasi-isotropic carbon/epoxy laminates with sharp and blunt notches. Compos Sci Technol 2014;100:220-7.

[7] Camanho PP, Maimí P, Dávila CG. Prediction of size effects in notched laminates using continuum damage mechanics. Compos Sci Technol 2007;67 (13):2715-27.

[8] Hallett SR, Green BG, Jiang WG, Wisnom MR. An experimental and numerical investigation into the damage mechanisms in notched composites. Compos A Appl Sci Manuf 2009;40(5):613-24.

[9] Whitney JM, Nuismer RJ. Stress fracture criteria for laminated composites containing stress concentrations. J Compos Mater 1974;8(3):253-65.

[10] Wang J, Callus P, Bannister M. Experimental and numerical investigation of the tension and compression strength of un-notched and notched quasi-isotropic laminates. Compos Struct 2004;64(3-4):297-306.

[11] Awerbuch J, Madhukar MS. Notched strength of composite laminates: predictions and experiments-a review. J Reinf Plast Compos 1985;4(1):3-159.

[12] Hochard C, Lahellec N, Bordreuil C. A ply scale non-local fibre rupture criterion for CFRP woven ply laminated structures. Compos Struct 2007;80 (3):321-6.

[13] Camanho PP, Erçin GH, Catalanotti G, Mahdi S, Linde P. A finite fracture mechanics model for the prediction of the open-hole strength of composite laminates. Compos A Appl Sci Manuf 2012;43(8):1219-25.

[14] Leguillon D, Quesada D, Putot C, Martin E. Prediction of crack initiation at blunt notches and cavities - size effects. Eng Fract Mech 2007;74 (15):2420-36.

[15] Mohammed Y, Hassan MK, El-Ainin HA, Hashem AM. Size effect analysis of open-hole glass fiber composite laminate using two-parameter cohesive laws. Acta Mech 2015;226(4):1027-44.

[16] Dugdale DS. Yielding of steel sheets containing slits. J Mech Phys Solids 1960;8 (2):100-4.

[17] Barenblatt GI. The mathematical theory of equilibrium cracks in brittle fracture. Adv Appl Mech 1962;7:55-129.

[18] Hallett SR. Experimental investigation of progressive damage and the effect of layup in notched tensile tests. J Compos Mater 2005;40(2):119-41.

[19] Hessabi ZR, Majidi B, Aghazadeh J. Effects of stacking sequence on fracture mechanisms in quasi-isotropic carbon/epoxy laminates under tensile loading. Iran Polym J 2005;14(6):531.
[20] Camanho PP, Hallett SR. Numerical modelling of failure in advanced composite materials. Woodhead Publishing: 2015.

[21] Hongkarnjanakul N. Modélisation numérique pour la tolérance aux dommages d'impact (PhD thesis). ISAE Supaéro; 2013. <http://depozit.isae.fr/theses/2013/ 2013_Hongkarnjanakul_Natthawat.pdf>.

[22] Chen BY, Tay TE, Baiz PM, Pinho ST. Numerical analysis of size effects on openhole tensile composite laminates. Compos A Appl Sci Manuf 2013;47:52-62.

[23] Abisset E, Daghia F, Ladevèze P. On the validation of a damage mesomodel for laminated composites by means of open-hole tensile tests on quasi-isotropic laminates. Compos A Appl Sci Manuf 2011;42(10):1515-24.

[24] Laurin F, Carrere N, Huchette C, Maire J-F. A multiscale hybrid approach for damage and final failure predictions of composite structures. J Compos Mater 2013;47(20-21):2713-47.

[25] Ridha M, Wang CH, Chen BY, Tay TE. Modelling complex progressive failure in notched composite laminates with varying sizes and stacking sequences. Compos A Appl Sci Manuf 2014;58:16-23.

[26] Wisnom MR. Modelling discrete failures in composites with interface elements. Compos A Appl Sci Manuf 2010;41(7):795-805.

[27] Xu X, Wisnom MR, Li X, Hallett SR. A numerical investigation into size effects in centre-notched quasi-isotropic carbon/epoxy laminates. Compos Sci Technol 2015;111:32-9.

[28] Sun XC, Wisnom MR, Hallett SR. Interaction of inter- and intralaminar damage in scaled quasi-static indentation tests: Part 2 - Numerical simulation. Compos Struct 2016;136:727-42.

[29] Aymerich F, Dore F, Priolo P. Prediction of impact-induced delamination in cross-ply composite laminates using cohesive interface elements. Compos Sci Technol 2008;68(12):2383-90.

[30] Lammerant L. Modelling of the interaction between matrix cracks and delaminations during impact of composite plates. Compos Sci Technol 1996;56(10):1171-8.

[31] de Moura MFS, Gonçalves JP. Modelling the interaction between matrix cracking and delamination in carbon-epoxy laminates under low velocity impact. Compos Sci Technol 2004;64(7-8):1021-7.

[32] Zhang Y, Zhu P, Lai X. Finite element analysis of low-velocity impact damage in composite laminated plates. Mater Des 2006;27(6):513-9.

[33] Geubelle PH, Baylor JS. Impact-induced delamination of composites: a 2D simulation. Compos Part B Eng 1998;29(5):589-602.

[34] Bouvet C, Castanié B, Bizeul M, Barrau J-J. Low velocity impact modelling in laminate composite panels with discrete interface elements. Int J Solids Struct 2009;46(14-15):2809-21.

[35] Bouvet C, Rivallant S, Barrau JJ. Low velocity impact modeling in composite laminates capturing permanent indentation. Compos Sci Technol 2012;72 (16): 1977-88.

[36] Rivallant S, Bouvet C. Hongkarnjanakul N. Failure analysis of CFRP laminates subjected to compression after impact: FE simulation using discrete interface elements. Compos A Appl Sci Manuf 2013;55:83-93.

[37] Adam L, Bouvet C, Castanié B, Daidié A, Bonhomme E. Discrete ply model of circular pull-through test of fasteners in laminates. Compos Struct 2012;94 (10):3082-91.

[38] Achard V, Bouvet C, Castanié B, Chirol C. Discrete ply modelling of open hole tensile tests. Compos Struct 2014;113:369-81.

[39] Pagano NJ, Pipes RB. The influence of stacking sequence on laminate strength. In: Mechanics of Composite Materials. Springer; 1994. p. 246-54.

[40] Grayley ME. Delamination and free edge stresses in composite laminates subjected to uniform prescribed axial strain and temperature changes. ESDU; 1995.

[41] N. Li and P. H. Chen, "Micro-macro FE modelling of damage evolution in laminated composite plates subjected to low velocity impact", Compos. Struct. on-line, doi:10.1016/j.compstruct.2016.02.063.

[42] Bažant ZP, Oh B. Progressive crack band theory for fracture of concrete. Mater Struct 1983:155-77.

[43] Cosserat E, Cosserat F. Théorie des corps déformables. Hermann et fils. Hermann et fils; 1909.

[44] Germain N. Modélisation non locale de l'endommagement dans les structures composites ( $\mathrm{PhD}$ thesis). École Nationale Supérieure des Mines de Paris: 2006.

[45] Allix O, Feissel P, Thévenet P. A delay damage mesomodel of laminates under dynamic loading: basic aspects and identification issues. Comput Struct 2003;81(12):1177-91.

[46] Marcin L, Maire J-F, Carrère N, Martin E. Development of a macroscopic damage model for woven ceramic matrix composites. Int J Damage Mech 2011;20(6):939-57.

[47] Hellweg H-B. Nonlinear failure simulation of thick composite structures ( $\mathrm{PhD}$ thesis). London: Imperial College of Science, Technology and Medicine; 1994.

[48] Pinho ST. Modelling failure of laminated composites using physically-based failure models (PhD thesis). Imperial College London; 2005.

[49] <http://www.institut-clement-ader.org/vertex/>.

[50] Lisle T, Bouvet C, Pastor ML, Margueres P, Prieto Corral R. Damage analysis and fracture toughness evaluation in a thin woven composite laminate under static tension using infrared thermography. Compos A Appl Sci Manuf 2013;53: 75-87.

[51] Laffan MJ, Pinho ST, Robinson P, Iannucci L. Measurement of the in situ ply fracture toughness associated with mode I fibre tensile failure in FRP. Part II: size and lay-up effects. Compos Sci Technol 2010;70(4):614-21.

[52] Sebaey TA, González EV, Lopes CS, Blanco N, Costa J. Damage resistance and damage tolerance of dispersed CFRP laminates: effect of ply clustering. Compos Struct 2013;106:96-103. 